\title{
Combinatorics of dense subsets of the rationals
}

\author{
by \\ B. Balcar (Praha), F. Hernández-Hernández (Morelia) and \\ M. Hrušák (Morelia)
}

\begin{abstract}
We study combinatorial properties of the partial order (Dense $(\mathbb{Q}), \subseteq$ ). To do that we introduce cardinal invariants $\mathfrak{p}_{\mathbb{Q}}, \mathfrak{t}_{\mathbb{Q}}, \mathfrak{h}_{\mathbb{Q}}, \mathfrak{s}_{\mathbb{Q}}, \mathfrak{r}_{\mathbb{Q}}, \mathfrak{i}_{\mathbb{Q}}$ describing properties of $\operatorname{Dense}(\mathbb{Q})$. These invariants satisfy $\mathfrak{p}_{\mathbb{Q}} \leq \mathfrak{t}_{\mathbb{Q}} \leq \mathfrak{h}_{\mathbb{Q}} \leq \mathfrak{s}_{\mathbb{Q}} \leq \mathfrak{r}_{\mathbb{Q}} \leq \mathfrak{i}_{\mathbb{Q}}$. We compare them with their analogues in the well studied Boolean algebra $\mathcal{P}(\omega) /$ fin. We show that $\mathfrak{p}_{\mathbb{Q}}=\mathfrak{p}, \mathfrak{t}_{\mathbb{Q}}=\mathfrak{t}$ and $\mathfrak{i}_{\mathbb{Q}}=\mathfrak{i}$, whereas $\mathfrak{h}_{\mathbb{Q}}>\mathfrak{h}$ and $\mathfrak{r}_{\mathbb{Q}}>\mathfrak{r}$ are both shown to be relatively consistent with ZFC. We also investigate combinatorics of the ideal nwd of nowhere dense subsets of $\mathbb{Q}$. In particular, we show that $\operatorname{non}(\mathcal{M})=\min \{|\mathcal{D}|: \mathcal{D} \subseteq \operatorname{Dense}(\mathbb{R}) \wedge(\forall I \in \operatorname{nwd}(\mathbb{R}))(\exists D \in \mathcal{D})$ $(I \cap D=\emptyset)\}$ and $\operatorname{cof}(\mathcal{M})=\min \{|\mathcal{D}|: \mathcal{D} \subseteq \operatorname{Dense}(\mathbb{Q}) \wedge(\forall I \in \operatorname{nwd})(\exists D \in \mathcal{D})(I \cap D=\emptyset)\}$. We use these facts to show that $\operatorname{cof}(\mathcal{M}) \leq \mathfrak{i}$, which improves a result of S. Shelah.
\end{abstract}

0. Introduction. The aim of this paper is to point out the similarities and differences between the structure of $\mathcal{P}(\omega)$ /fin and the structure of the collection of dense subsets of the rationals. Such research was suggested by A. Blass in [Bl] and initiated by J. Cichoń in [Ci]. The basic object studied here is the set

$$
\operatorname{Dense}(\mathbb{Q})=\{D \subseteq \mathbb{Q}: D \text { is dense }\}
$$

ordered by inclusion, in comparison with the structure $\left([\omega]^{\omega}, \subseteq\right)$. Neither one of them is a separative partial order. The separative quotient of $\left([\omega]^{\omega}, \subseteq\right)$ augmented with the least element 0 is the well known Boolean algebra $\mathcal{P}(\omega) /$ fin. The separative quotient of $(\operatorname{Dense}(\mathbb{Q}), \subseteq)$ with added least element is not a Boolean algebra, but just a lattice, with two dense sets being in the same equivalence class if and only if their symmetric difference is a

2000 Mathematics Subject Classification: 03E17, 03E35, 06E15.

Key words and phrases: rational numbers, nowhere dense ideal, distributivity of Boolean algebras, cardinal invariants of the continuum.

The research of the first and third authors was partially supported by grant GAČR 201/03/0933. The first author's research was also partially supported by grant GAČR 201/02/0857 and that of the third author was supported by PAPIIT grant IN108802-2 and CONACYT grant 40057-F. 
nowhere dense subset of $\mathbb{Q}$. It is convenient not to study the quotients directly but rather to use a pre-order $\subseteq^{*}$ in the case of $\mathcal{P}(\omega) /$ fin and $\subseteq$ nwd in the case of Dense $(\mathbb{Q})$, where nwd denotes the ideal of nowhere dense subsets of $\mathbb{Q}$.

Whereas the structure of $\mathcal{P}(\omega)$ /fin and related cardinal invariants seem to be now fairly well understood and certainly well studied, the same can hardly be said about the next natural step - the study of combinatorial properties of subsets of the rational numbers $\mathbb{Q}$. The hope is that having at least a basic theory at hand (which this note humbly presents) new applications will arise.

We consider some cardinal invariants of $\left(\operatorname{Dense}(\mathbb{Q}), \subseteq_{\text {nwd }}\right)$ and compare them with their $\mathcal{P}(\omega) /$ fin analogues. Our aim was not to introduce more cardinal invariants, however, introducing them seemed necessary for understanding the similarities and differences between the structural properties of $\mathcal{P}(\omega) /$ fin and Dense $(\mathbb{Q})\left({ }^{1}\right)$.

1. Basic facts and definitions. The rationals $\mathbb{Q}$ were characterized by W. Sierpiński (see $[\mathrm{Kr}]$ ) as the unique (up to homeomorphism) countable first countable regular space without isolated points. Consider the set $2^{<\omega}$ of all finite sequences of 0 's and 1's. The set $2^{<\omega}$ ordered by extension (reverse inclusion) is a partially ordered set known as Cohen forcing. For our purposes it will be useful to give $2^{<\omega}$ the structure of $\mathbb{Q}$ in the following way: For $s, t \in 2^{<\omega}$ define $s<t$ if and only if $(t \subset s$ and $s(|t|)=0)$ or $(s \subset t$ and $t(|s|)=1)$ or $(k=\min \{n \in \operatorname{dom}(s) \cap \operatorname{dom}(t): s(n) \neq t(n)\}$ exists and $s(k)=0)$. This ordering is a linear ordering on $2^{<\omega}$ which induces a topology on $2^{<\omega}$ homeomorphic to $\mathbb{Q}$. We will identify $\mathbb{Q}$ with the set $2^{<\omega}$ endowed with this topology. Given $s \in 2^{<\omega}$ denote by $B_{s}$ the set $\left\{t \in 2^{<\omega}: s \subseteq t\right\}$.

We will compare the topological structure of $\mathbb{Q}=\left(2^{<\omega},<\right)$ with the usual partial order structure $\left(2^{<\omega}, \supseteq\right)$. Any mention of antichains in $2^{<\omega}$ refers to antichains in $\left(2^{<\omega}, \supseteq\right)$.

FACT 1.1. (i) The family $\left\{B_{s}: s \in 2^{<\omega}\right\}$ forms a $\pi$-base in $\mathbb{Q}=\left(2^{<\omega},<\right)$.

(ii) $D \subseteq 2^{<\omega}$ is dense in $\mathbb{Q}$ if and only if $D$ is dense in the partial order $\left(2^{<\omega}, \supseteq\right)$, i.e. for every $s \in 2^{<\omega}$ there is a $t \in D$ with $s \subseteq t$.

(iii) $D \subseteq 2^{<\omega}$ is dense in $\mathbb{Q}$ if and only if $D=\bigcup_{n \in \omega} A_{n}$, where each $A_{n}$ is a maximal antichain in $\left(2^{<\omega}, \supseteq\right)$ such that $\left|\left\{t \in A_{n+1}: s \subseteq t\right\}\right| \geq 2$ for every $s \in A_{n}$.

(iv) $H \subseteq 2^{<\omega}$ is open dense in $\mathbb{Q}$ then $H$ contains a subset $H^{\prime}$ which is open dense in the partial order $\left(2^{<\omega}, \supseteq\right)$, i.e. $H^{\prime}$ is dense and

$\left(^{1}\right)$ "People know what they do. They generally know why they do what they do. What they don't know is what what they do does" (M. Foucault, Dreyfuss and Rabinow). 
$B_{s} \subseteq H^{\prime}$ for every $s \in H^{\prime}$. On the other hand, if $H$ is open dense in $\left(2^{<\omega}, \supseteq\right)$ then $H$ is open dense in $\mathbb{Q}$.

(v) $N \subseteq 2^{<\omega}$ is nowhere dense in $\mathbb{Q}$ if and only if $\left(\forall s \in 2^{<\omega}\right)(\exists t \supseteq s)$ $\left(B_{t} \cap N=\emptyset\right)$.

Note that $N \subseteq 2^{<\omega}$ is nowhere dense if and only if there is a maximal antichain $A \subseteq 2^{<\omega}$ such that $(\forall s \in N)(\exists t \in A)(s \subseteq t)$. Moreover, if $N \subseteq 2^{<\omega}$ is nowhere dense then the closure of $N$ under initial segments, i.e. the set $\left\{t \in 2^{<\omega}:(\exists s \in N)(t \subseteq s)\right\}$, is a closed nowhere dense set containing $N$.

There is a natural correspondence between the structure of $2^{<\omega}$ and the topology of the Cantor space $2^{\omega}$. For $s \in 2^{<\omega}$ we denote by $\langle s\rangle$ the basic clopen set $\left\{f \in 2^{\omega}: s \subseteq f\right\}$ of $2^{\omega}$. Given a subset $X$ of $2^{<\omega}$ let $\widehat{X}=\left\{f \in 2^{\omega}:\left(\exists^{\infty} n \in \omega\right)(f\lceil n \in X)\}\right.$. This is a $G_{\delta}$ subset of $2^{\omega}$. For $K \subseteq 2^{\omega}$ let $\widetilde{K}=\{f\lceil n: f \in K$ and $n \in \omega\}$.

FACT 1.2. (i) $X \subseteq 2^{<\omega}$ is dense if and only if $\widehat{X}$ is dense $G_{\delta}$ in $2^{\omega}$.

(ii) If $X \subseteq 2^{<\omega}$ is dense open then $\widehat{X}$ is dense open in $2^{\omega}$.

(iii) Let $X \subseteq 2^{<\omega}$ be closed under initial segments. If, moreover, $X$ is nowhere dense in $2^{<\omega}$ then $\widehat{X}$ is closed nowhere dense in $2^{\omega}$. Moreover, $X$ is an infinite closed nowhere dense subset of $\mathbb{Q}$ if and only if $\widehat{X}$ is a non-empty closed nowhere dense set.

(iv) If $Y$ is a dense $G_{\delta}$ subset of $2^{\omega}$ then there is a (non-unique) dense $X \subseteq 2^{<\omega}$ such that $Y=\widehat{X}$.

(v) If $\bar{K}$ is a closed nowhere dense subset of $2^{\omega}$ then $N=\widetilde{K}$ is closed nowhere dense in $\mathbb{Q}$ and $K=\widehat{N}$.

Proof. All but (iv) are completely straightforward. To prove (iv) write $Y$ as the intersection of a decreasing sequence of $V_{n}, n \in \omega$, where each $V_{n}$ is open dense. By zero-dimensionality of $2^{\omega}$ each $V_{n}$ can be written as a disjoint union of clopen sets, i.e. $V_{n}=\bigcup\left\{\left\langle s_{i}\right\rangle: i \in I_{n}\right\}$, where $A_{n}=\left\{s_{i}: i \in I_{n}\right\}$ is a maximal antichain in $2^{<\omega}$. Without loss of generality $A_{n+1}$ refines $A_{n}$. Put $X=\bigcup\left\{A_{n}: n \in \omega\right\}$.

Recall the definitions and basic facts about the relevant cardinal invariants of the continuum (see e.g. $[\mathrm{BJ}]$ ). The symbol $\mathfrak{b}$ denotes the unbounding number of $\left(\omega^{\omega}, \leq^{*}\right)$ and $\mathfrak{d}$ denotes the dominating number of $\left(\omega^{\omega}, \leq^{*}\right)$.

$\operatorname{cov}(\mathcal{M})$ is the minimal size of a family of meager subsets of $2^{\omega}$ that cover $2^{\omega}$, and $\operatorname{add}(\mathcal{M})$ stands for the additivity of the meager ideal, i.e. the minimal size of a family of meager subsets of $2^{\omega}$ whose union is not meager. $\operatorname{non}(\mathcal{M})$ is the minimal size of a non-meager subset of $2^{\omega}$, and $\operatorname{cof}(\mathcal{M})$ is the minimal size of a cofinal family of meager subsets of $2^{\omega}$. It is easy to see that $\operatorname{add}(\mathcal{M})$ and $\operatorname{cof}(\mathcal{M})$ are uncountable regular cardinals. The cardinal invariants $\mathfrak{p}, \mathfrak{t}$ and $\mathfrak{h}$ are defined later on in the text. 
The following proposition sums up provable relationships between these cardinal invariants:

Proposition 1.3. (i) $\mathfrak{p} \leq \mathfrak{t} \leq \mathfrak{h} \leq \mathfrak{b} \leq \mathfrak{d}$ and $\mathfrak{b} \leq \operatorname{non}(\mathcal{M})$,

(ii) (Piotrowski, Szymański) $\mathfrak{t} \leq \operatorname{add}(\mathcal{M})$,

(iii) (Truss, Miller) $\operatorname{add}(\mathcal{M})=\min \{\mathfrak{b}, \operatorname{cov}(\mathcal{M})\}$,

(iv) (Fremlin) $\operatorname{cof}(\mathcal{M})=\max \{\mathfrak{d}, \operatorname{non}(\mathcal{M})\}$.

The invariants $\mathfrak{h}$ and $\operatorname{add}(\mathcal{M})$ as well as $\mathfrak{b}$ and $\operatorname{cov}(\mathcal{M})$ are not provably comparable in ZFC. For proofs and additional information consult $[\mathrm{BJ}]$ and $[\mathrm{BS}]$; see also $[\mathrm{Va}]$. The following reformulation of a result of $\mathrm{K}$. Keremedis $([\mathrm{Ke}])$ is the key to the study of the structure of the rationals. For the sake of completeness and also since the published proofs ([Ke], [BJ]) are quite technical and (at least in our opinion) hard to follow, we present a direct proof here.

THEOREM 1.4 (Keremedis). (i) $\operatorname{cov}(\mathcal{M})$ is equal to the minimal size of a family $\mathcal{F}$ of nowhere dense subsets of $\mathbb{Q}$ such that for every $Y \in[\mathbb{Q}]^{\omega}$ there is an $F \in \mathcal{F}$ intersecting $Y$ in an infinite set.

(ii) $\operatorname{add}(\mathcal{M})$ is equal to the minimal size of a family $\mathcal{F}$ of nowhere dense subsets of $\mathbb{Q}$ such that for every $D \in \operatorname{Dense}(\mathbb{Q})$ there is an $F \in \mathcal{F}$ intersecting $D$ in an infinite set.

Proof. (i) For the purposes of this proof we denote by $\mu$ the minimal size of a family $\mathcal{F}$ of nowhere dense subsets of $\mathbb{Q}$ such that for every $Y \in[\mathbb{Q}]^{\omega}$ there is an $F \in \mathcal{F}$ intersecting $Y$ in an infinite set. We divide the proof into three parts:

(1) $\operatorname{cov}(\mathcal{M}) \leq \mu$,

(2) $\mu \leq \mathfrak{d}$,

(3) $\mu \leq \operatorname{cov}(\mathcal{M})$.

For (1) fix $\kappa<\operatorname{cov}(\mathcal{M})$ and let $\left\{F_{\alpha}: \alpha<\kappa\right\}$ be a family of nowhere dense subsets of $2^{<\omega}$, closed under initial segments. As $\kappa<\operatorname{cov}(\mathcal{M})$, there is an $r \in 2^{\omega} \backslash \bigcup\left\{\widehat{F}_{\alpha}: \alpha<\kappa\right\}$. Recall that $\widehat{F}_{\alpha}$ was introduced in Section 1. Put $Y=\left\{r\lceil n: n \in \omega\}\right.$. Then $Y \cap F_{\alpha}$ is finite for all $\alpha<\kappa$.

In order to prove (2), fix three disjoint countable dense subsets $\mathbb{Q}, D_{1}$, $D_{2}$ of $2^{\omega}$ and a strictly (not mod fin) dominating family $\left\{f_{\alpha}: \alpha<\mathfrak{d}\right\}$ in $\omega^{\omega}$ consisting of functions with non-zero values. Enumerate each $D_{i}$ as $\left\{d_{n}^{i}: n \in \omega\right\}$. For $\alpha<\mathfrak{d}$ put

$$
O_{\alpha}=\bigcup_{n \in \omega} B\left(d_{n}^{1}, 1 / f_{\alpha}(n)\right) \cap \bigcup_{n \in \omega} B\left(d_{n}^{2}, 1 / f_{\alpha}(n)\right),
$$

where $B(x, 1 / n)$ denotes the ball in $2^{\omega}$ of radius $1 / n$ centred at $x$. Note that each $O_{\alpha}$ is a dense open subset of $2^{\omega}$; hence $N_{\alpha}=\mathbb{Q} \backslash O_{\alpha}$ is a nowhere dense subset of $\mathbb{Q}$. Aiming toward a contradiction assume that there is $Y \in[\mathbb{Q}]^{\omega}$ 
intersecting each $N_{\alpha}$ in a finite set. Then, obviously, any infinite subset of $Y$ has the same property. Pick an infinite subset $Y_{0}$ of $Y$ converging to some $r \in 2^{\omega}$. Then either $r \notin D_{1}$ or $r \notin D_{2}$, say $r \notin D_{1}$. The set $Y_{1}=Y_{0} \cup\{r\}$ is then a compact subset of $2^{\omega}$ disjoint from $D_{1}$. Consider the distances of points $d_{n}^{1}$ to $Y_{1}$. Obviously all are positive, so there is an $\alpha<\mathfrak{d}$ such that $\operatorname{dist}\left(d_{n}^{1}, Y_{1}\right) \geq 1 / f_{\alpha}(n)$ for all $n \in \omega$. This, however, means that $Y_{0} \subseteq N_{\alpha}$, which is a contradiction.

For (3), fix $\kappa<\mu$ and let $\left\{F_{\alpha}: \alpha<\kappa\right\}$ be a family of closed nowhere dense subsets of $2^{\omega}$. By Fact $1.2,\left\{\widetilde{F}_{\alpha}: \alpha<\kappa\right\}$ is then a family closed nowhere dense subsets of $\mathbb{Q}=\left(2^{<\omega},<\right)$. As $\kappa<\mu$, there is a set $Y \in\left[2^{<\omega}\right]^{\omega}$ having finite intersection with all $\widetilde{F}_{\alpha}$. To finish the proof it suffices to prove the following.

Claim. There is a $Y$ forming a decreasing chain in $\left(2^{<\omega}, \supseteq\right)$.

If there were such $Y$, then the real $r=\bigcup\{s: s \in Y\} \in 2^{\omega}$ would not be covered by $\bigcup\left\{F_{\alpha}: \alpha<\kappa\right\}$.

We prove the Claim by contradiction. First notice that if $Y$ is infinite and does not contain an infinite chain it contains an infinite antichain. Recursively construct an infinite sequence of antichains $A_{n}$ in $2^{<\omega}$ so that:

(a) $A_{n}$ intersects each $\widetilde{F}_{\alpha}$ in a finite set,

(b) $A_{n+1}$ refines $A_{n}$,

(c) for every $s \in A_{n}$ there are infinitely many $t \in A_{n+1}$ such that $s \subset t$.

To start, let $A_{0}$ be an arbitrary infinite antichain satisfying (a). Having constructed $A_{n}$, look at $B_{s}$ for $s \in A_{n}$. It is an open subset of $\left(2^{<\omega},<\right)$ homeomorphic to $\mathbb{Q}$, so by the hypothesis, there is an infinite antichain $A^{s} \subseteq B_{s}$ intersecting each $\widetilde{F}_{\alpha}$ in a finite set. Set

$$
A_{n+1}=\bigcup\left\{A^{s}: s \in A_{n}\right\} \text {. }
$$

Note that, as $\widetilde{F}_{\alpha}$ is upward closed, $\widetilde{F}_{\alpha} \cap A_{n}$ is finite for every $n \in \omega$. Enumerate each $A_{n}$ as $\left\{s_{i}^{n}: i \in \omega\right\}$ and let, for $\alpha<\kappa$,

$$
g_{\alpha}(n)=\min \left\{k \in \omega:(\forall m \geq k)\left(s_{m}^{n} \notin \widetilde{F}_{\alpha}\right)\right\} .
$$

As $\kappa<\mu \leq \mathfrak{d}$ there is a function $g$ which is not $\leq^{*}$-dominated by any $g_{\alpha}$, $\alpha<\kappa$. Now, recursively construct a decreasing sequence $\left\{t_{n}: n \in \omega\right\} \subseteq 2^{<\omega}$ so that:

(d) $t_{n} \in A_{n}$,

(e) $t_{n}=s_{i}^{n}$ for some $i>g(n)$.

Now, for each $\alpha<\kappa$ there is an $n \in \omega$ such that $t_{n} \notin \widetilde{F}_{\alpha}$. However, as $\widetilde{F}_{\alpha}$ is upward closed, this means that $t_{m} \notin \widetilde{F}_{\alpha}$ for all $m \geq n$. So $Y=\left\{t_{n}: i \in \omega\right\}$ 
is a decreasing chain in $2^{<\omega}$ intersecting each $\widetilde{F}_{\alpha}$ in a finite set, which is a contradiction and the proof of the Claim is finished.

(ii) Denote by $\mu_{D}$ the minimal size of a family $\mathcal{F}$ of nowhere dense subsets of $\mathbb{Q}$ such that for every $D \in \operatorname{Dense}(\mathbb{Q})$ there is an $F \in \mathcal{F}$ intersecting $D$ in an infinite set.

To see that $\operatorname{add}(\mathcal{M}) \leq \mu_{D}$, fix $\kappa<\operatorname{add}(\mathcal{M})$ and let $\left\{F_{\alpha}: \alpha<\kappa\right\}$ be a family of nowhere dense subsets of $\mathbb{Q}$. Fix also an enumeration $\left\{W_{n}: n \in \omega\right\}$ of a basis for the topology on $\mathbb{Q}$. By part (i) of the theorem and the fact that $\kappa<\operatorname{add}(\mathcal{M}) \leq \operatorname{cov}(\mathcal{M})$, for every $W_{n}$ there is an infinite set $Y_{n}=\left\{y_{i}^{n}: i \in \omega\right\}$ $\subseteq W_{n}$ intersecting each $F_{\alpha}$ in a finite set. Put

$$
g_{\alpha}(n)=\min \left\{k \in \omega:(\forall m \geq k)\left(y_{m}^{n} \notin F_{\alpha}\right)\right\} .
$$

As $\kappa<\operatorname{add}(\mathcal{M}) \leq \mathfrak{b}$ there is a function $g$ dominating all $g_{\alpha}, \alpha<\kappa$. Set $D=\left\{y_{m}^{n}: n \in \omega\right.$ and $\left.m \geq g(n)\right\}$. This is obviously a dense set as it intersects each $W_{n}$ in an infinite set. Moreover, as $g$ dominates $g_{\alpha},\left\{y_{m}^{n}\right.$ : $m \geq g(n)\} \cap F_{\alpha}=\emptyset$ for all but finitely many $n$; hence $D \cap F_{\alpha}$ is finite, being a finite union of finite sets.

For the other direction, fix $\kappa<\mu_{D}$ and let $\left\{N_{\alpha}: \alpha<\kappa\right\}$ be a family of closed nowhere dense subsets of $2^{\omega}$. The sets $\widetilde{N}_{\alpha}$ are then nowhere dense in $\mathbb{Q}=\left(2^{<\omega},<\right)$, so there is a dense set $D \subseteq 2^{<\omega}$ such that $D \cap \widetilde{N}_{\alpha}$ is finite for every $\alpha<\kappa$. Then $\widehat{D}$ is a dense $G_{\delta}$ subset of $2^{\omega}$ disjoint from all $N_{\alpha}$. Therefore $\bigcup_{\alpha<\kappa} N_{\alpha}$ is a meager set, thus $\kappa<\operatorname{add}(\mathcal{M})$.

Now we establish results in some sense dual to Keremedis' Theorem. The equality $\operatorname{cof}(\mathcal{M})=\operatorname{cof}($ nwd $)$ was already proven by Fremlin in $[\mathrm{Fr}]$; we give a new and simpler proof. First, we make a simple observation.

FACT 1.5. If $X$ is a separable metric space without isolated points, then

$$
\operatorname{cof}(\operatorname{nwd}(X))=\operatorname{cof}(\text { nwd }) \text {. }
$$

Indeed, if $X$ is a separable metric space without isolated points and $D$ is a countable dense subset of $X$, it is a classical fact that $D$ is homeomorphic to $\mathbb{Q}$. Now, it will suffice to show that given any closed $F \in \operatorname{nwd}(X)$, there is $N \in \operatorname{nwd}(D)$ so that $F \subseteq \bar{N}$. Write $F=\bigcap_{n \in \omega} U_{n}$ with $U_{n}$ open and $U_{n} \supset \bar{U}_{n+1}$ for each $n \in \omega$. Choosing a maximal subset of $D \cap\left(U_{n} \backslash \bar{U}_{n+1}\right)$ such that any two of its elements are at distance at least $1 /(n+1)$, for each $n \in \omega$, and taking as $N$ the union of those subsets will do the job.

THEOREM 1.6. (i) $\operatorname{cof}(\mathcal{M})=\operatorname{cof}($ nwd) and they are equal to the minimal size of a family $\mathcal{D}$ of dense subsets of $\mathbb{Q}$ such that for every nowhere dense $I \subseteq \mathbb{Q}$ there is $D \in \mathcal{D}$ disjoint from $I$.

(ii) $\operatorname{non}(\mathcal{M})$ is equal to the minimal size of a family $\mathcal{D}$ of dense subsets of $\mathbb{R}$ such that for every nowhere dense $I \subseteq \mathbb{R}$ there is $D \in \mathcal{D}$ disjoint from $I$. 
We will prove both clauses of the theorem almost simultaneously using the following lemmas. For the purpose of the proof let

$$
\begin{aligned}
& \mu_{\mathbb{Q}}=\min \{|\mathcal{D}|: \mathcal{D} \subseteq \operatorname{Dense}(\mathbb{Q}) \wedge(\forall I \in \operatorname{nwd})(\exists D \in \mathcal{D})(I \cap D=\emptyset)\}, \\
& \mu_{\mathbb{R}}=\min \{|\mathcal{D}|: \mathcal{D} \subseteq \operatorname{Dense}(\mathbb{R}) \wedge(\forall I \in \operatorname{nwd}(\mathbb{R}))(\exists D \in \mathcal{D})(I \cap D=\emptyset)\} .
\end{aligned}
$$

LEMMA 1.7. Let $M$ be a meager set, let $\kappa<\mathfrak{d}$, and for each $\alpha<\kappa$, let $D_{\alpha} \subseteq \mathbb{R}$ be such that $D_{\alpha} \cap M$ is dense in $\mathbb{R}$. Then there exists $N \subseteq M$ which is nowhere dense and such that $D_{\alpha} \cap N \neq \emptyset$ for every $\alpha<\kappa$.

Proof. Let $U_{m}, m \in \omega$, be a family of pairwise disjoint non-empty intervals in $\mathbb{R}$. Note that $D_{\alpha} \cap M$ is dense in $U_{m}$ for every $\alpha<\kappa$ and $m \in \omega$. Now, for every $m \in \omega$, there are nowhere dense sets $\left\{F_{n}^{m}: n \in \omega\right\}$ such that $M \cap U_{m}=\bigcup_{n \in \omega} F_{n}^{m}$. Put $F_{n}=\bigcup_{m \in \omega} F_{n}^{m}$. The sets $F_{n}$ are also nowhere dense, and moreover $F_{n} \cap U_{m} \neq \emptyset$ for every $m, n \in \omega$. For each $\alpha<\kappa$ define $f_{\alpha}: \omega \rightarrow \omega$ by

$$
f_{\alpha}(n)=\min \left\{k \in \omega: D_{\alpha} \cap U_{n} \cap F_{k} \neq \emptyset\right\}+1 .
$$

As $\kappa<\mathfrak{d}$, there is a $g: \omega \rightarrow \omega$ such that $g \not^{*} f_{\alpha}$ for every $\alpha<\kappa$. Then define

$$
N=\bigcup_{n \in \omega}\left\{F_{k} \cap U_{n}: k<g(n)\right\} .
$$

Clearly $N$ is nowhere dense and given $\alpha<\kappa$, there is an $n \in \omega$ such that $f_{\alpha}(n)<g(n)$. Therefore $D_{\alpha} \cap U_{n} \cap F_{f_{\alpha}(n)} \neq \emptyset$, and so $D_{\alpha} \cap N \neq \emptyset$.

LEMMA 1.8. Let $M \subseteq 2^{\omega}$ be a meager set. Then there exists a nowhere dense $N$ such that $N^{*} \supseteq M$, where $N^{*}=\left\{f \in 2^{\omega}:(\exists g \in N)\left(f={ }^{*} g\right)\right\}$.

Proof. Let $M=\bigcup_{n \in \omega} F_{n}$, where $F_{n}$ is a nowhere dense subset of $2^{\omega}$. For each $n \in \omega$, consider the sequence $s_{n}$ starting with $n$ zeros followed by one 1 . Then define $F_{n}^{\prime}$ by: $t \in F_{n}^{\prime}$ if and only if $s_{n} \subseteq t \wedge\left(\exists u \in 2^{n+1}\right)(u \frown t \uparrow[n+1, \omega)$ $\left.\in F_{n}\right)$. Then, clearly, $F_{n}^{\prime}$ is nowhere dense and if $N=\bigcup_{n \in \omega} F_{n}^{\prime}$, then $N$ is nowhere dense and $M \subseteq N^{*}$.

LEMMA 1.9. $\operatorname{cof}(\mathcal{M}) \leq \max \left\{\mathfrak{d}, \mu_{\mathbb{R}}\right\}$.

Proof. Let $\left\{D_{\alpha}: \alpha<\mu_{\mathbb{R}}\right\}$ be a family of dense subsets of $\mathbb{R}$ witnessing the definition of $\mu_{\mathbb{R}}$, and let $\left\{f_{\beta}: \beta<\mathfrak{d}\right\}$ be a $\leq$-dominating family. Without loss of generality we can assume that all $D_{\alpha}$ are countable. Denote by $B(x, \varepsilon)$ the ball with centre at $x$ and radius $\varepsilon>0$. Fix an enumeration $\left\{d_{\alpha, n}: n \in \omega\right\}$ of $D_{\alpha}$ for each $\alpha<\mu_{\mathbb{R}}$. Then, for $\alpha<\mu_{\mathbb{R}}$ and $\beta<\mathfrak{d}$, define nowhere dense sets $N_{\alpha, \beta}$ by

$$
N_{\alpha, \beta}=\mathbb{R} \backslash \bigcup_{n \in \omega} B\left(d_{\alpha, n}, \frac{1}{f_{\beta}(n)+1}\right) .
$$

We claim that the family of all $N_{\alpha, \beta}^{*}$ is cofinal in $\mathcal{M}$. Indeed, if $M \subseteq \mathbb{R}$ is a meager set, then by Lemma 1.8, there exists a nowhere dense subset $N$ such 
that $M \subseteq N^{*}$. Thus it suffices to show that $N \subseteq N_{\alpha, \beta}$ for some $\alpha<\mu_{\mathbb{R}}$ and some $\beta<\mathfrak{d}$, as we will then have $N^{*} \subseteq N_{\alpha, \beta}^{*}$.

As $N$ is nowhere dense, there is an $\alpha<\mu_{\mathbb{R}}$ such that $D_{\alpha} \cap N=\emptyset$. Define $g: \omega \rightarrow \omega$ by

$$
g(n)=\min \left\{k \in \omega: B\left(d_{\alpha, n}, \frac{1}{k+1}\right) \cap N=\emptyset\right\} .
$$

As $\left\{f_{\beta}: \beta<\mathfrak{d}\right\}$ is dominating, there is $\beta<\mathfrak{d}$ such that $(\forall n \in \omega)(g(n) \leq$ $\left.f_{\beta}(n)\right)$. Thus $\mathbb{R} \backslash N_{\alpha, \beta} \subseteq \mathbb{R} \backslash N$.

We can now prove the theorem:

Proof of Theorem 1.6. First we prove that $\mathfrak{d} \leq \mu_{\mathbb{Q}}$. Fix $\kappa<\mathfrak{d}$. Let $\left\{D_{\alpha}\right.$ : $\alpha<\kappa\}$ be a family of dense subsets of $\mathbb{Q}$. Take $M=\mathbb{Q}$ and apply Lemma 1.7 with this family of dense subsets to infer the existence of a nowhere dense $N \subseteq \mathbb{Q}$ such that $D_{\alpha} \cap N \neq \emptyset$ for each $\alpha<\kappa$. Therefore $\kappa<\mu_{\mathbb{Q}}$.

It is trivial that $\operatorname{cof}(\mathrm{nwd}) \geq \mu_{\mathbb{Q}}$. To see that $\operatorname{cof}(\mathrm{nwd}) \leq \mu_{\mathbb{Q}}$ proceed as follows. Let $\left\{D_{\alpha}: \alpha<\mu_{\mathbb{Q}}\right\}$ be a family of dense subsets of $\mathbb{Q}$ witnessing the definition of $\mu_{\mathbb{Q}}$, each $D_{\alpha}$ enumerated as $\left\{d_{\alpha, n}: n \in \omega\right\}$, and consider a $\leq$-dominating family $\left\{g_{\beta}: \beta<\mathfrak{d}\right\}$; then define

$$
N_{\alpha, \beta}=\mathbb{Q} \backslash \bigcup_{n \in \omega} B\left(d_{\alpha, n}, \frac{1}{g_{\beta}(n)+1}\right) .
$$

This is a nowhere dense subset of $\mathbb{Q}$ and since we already know that $\mathfrak{d} \leq \mu_{\mathbb{Q}}$ we have $\mu_{\mathbb{Q}}$ such subsets. Moreover, if $N$ is any nowhere dense subset of $\mathbb{Q}$, there is some $\alpha<\mu_{\mathbb{Q}}$ such that $D_{\alpha} \cap \bar{N}=\emptyset$. Then define $g: \omega \rightarrow \omega$ by

$$
g(n)=\min \left\{k \in \omega: B\left(d_{\alpha, n}, \frac{1}{k+1}\right) \cap \bar{N}=\emptyset\right\} .
$$

There must exist $\beta<\mathfrak{d}$ such that $g \leq g_{\beta}$ and it follows that $N \subseteq N_{\alpha, \beta}$. Hence, $\left\{N_{\alpha, \beta}: \alpha<\mu_{\mathbb{Q}} \wedge \beta<\mathfrak{d}\right\}$ is a cofinal family in nwd.

Now, by Fact 1.5 together with Lemma 1.8, it follows that $\operatorname{cof}(\mathcal{M}) \leq$ $\operatorname{cof}(\mathrm{nwd})$. Likewise, Lemma 1.9 shows that $\operatorname{cof}(\mathcal{M}) \leq \mu_{\mathbb{Q}}$ since trivially $\mu_{\mathbb{R}} \leq \mu_{\mathbb{Q}}$. This completes the proof of the first part of the theorem.

To prove the second part, first notice that

$$
\min \{\mathfrak{d}, \operatorname{non}(\mathcal{M})\} \leq \mu_{\mathbb{R}} .
$$

This follows from Lemma 1.7. Indeed, assume $\kappa<\min \{\mathfrak{d}, \operatorname{non}(\mathcal{M})\}$ and let $\left\{D_{\alpha}: \alpha<\kappa\right\}$ be a family of countable dense subsets of $\mathbb{R}$. Then $M=$ $\bigcup_{\alpha<\kappa} D_{\alpha}$ is meager and by Lemma 1.7 there exists a nowhere dense $N \subseteq M$ such that $N \cap D_{\alpha} \neq \emptyset$ for every $\alpha<\kappa$. Thus $\kappa<\mu_{\mathbb{R}}$.

Secondly, $\mu_{\mathbb{R}} \leq \operatorname{non}(\mathcal{M})$. For suppose $\kappa<\mu_{\mathbb{R}}$ and consider $X \subseteq \mathbb{R}$ of cardinality $\kappa$. Define $D_{x}=x+\mathbb{Q}$. The family $\left\{D_{x}: x \in X\right\}$ cannot witness the definition of $\mu_{\mathbb{R}}$, therefore there must exist $F \in \operatorname{nwd}(\mathbb{R})$ such 
that $F \cap D_{x} \neq \emptyset$ for each $x \in X$. Notice that $F+\mathbb{Q} \supseteq X$. Thus $X$ is meager, and hence $\kappa<\operatorname{non}(\mathcal{M})$.

To finish the proof, consider two cases. If $\operatorname{non}(\mathcal{M}) \leq \mathfrak{d}$, then it follows that $\operatorname{non}(\mathcal{M}) \leq \mu_{\mathbb{R}} \leq \operatorname{non}(\mathcal{M})$. If it is not the case that non $(\mathcal{M}) \leq \mathfrak{d}$, then part (iii) of Proposition 1.3 implies that $\operatorname{cof}(\mathcal{M})=\operatorname{non}(\mathcal{M})$ and Lemma 1.9 now reads $\operatorname{non}(\mathcal{M}) \leq \max \left\{\mathfrak{d}, \mu_{\mathbb{R}}\right\}$, which together with $\mu_{\mathbb{R}} \leq \operatorname{non}(\mathcal{M})$ shows again that $\operatorname{non}(\mathcal{M})=\mu_{\mathbb{R}}$.

2. Dense $(\mathbb{Q})$ and $\mathcal{P}(\omega) /$ fin seem similar. In this section we point out the similarities between the structures $\mathcal{P}(\omega) /$ fin and Dense $(\mathbb{Q})$. In particular we show that some of their natural cardinal characteristics have the same value. First recall the following proposition mentioned in the introduction. For the rest of the paper $\left\{U_{n}: n \in \omega\right\}$ denotes a fixed enumeration of a basis for the topology on $\mathbb{Q}$.

Proposition 2.1. The separative modification of the order (Dense $(\mathbb{Q})$, $\subseteq)$ is the pre-order $(\operatorname{Dense}(\mathbb{Q}), \subseteq$ nwd $)$.

Proof. Note that $C, D \in \operatorname{Dense}(\mathbb{Q})$ are incompatible in $(\operatorname{Dense}(\mathbb{Q}), \subseteq)$ if and only if there is $n \in \omega$ such that $C \cap D \cap U_{n}=\emptyset$. It suffices to show that $C \subseteq$ nwd $D$ if and only if for every $X \in \operatorname{Dense}(\mathbb{Q}), X \cap C \in \operatorname{Dense}(\mathbb{Q})$ implies that $X \cap D \in \operatorname{Dense}(\mathbb{Q})$, as the condition on the right hand side defines the separative modification. Let $N=C \backslash D$ be nowhere dense in $\mathbb{Q}$. If $X \cap C$ is dense then $X \cap D \supseteq(X \cap C) \backslash(X \cap N)$ is also dense. On the other hand, if $C \backslash D$ is not nowhere dense then there is $n \in \omega$ such that $(C \backslash D) \cap U_{n}$ is dense in $U_{n}$. Put $X=\left(U_{n} \cap(C \backslash D)\right) \cup\left(C \backslash U_{n}\right)$. Then $X$ is dense, $X \subseteq C$ and $X$ is incompatible to $D$.

Proposition 2.2. The pre-order $\left(\operatorname{Dense}(\mathbb{Q}), \subseteq_{\text {nwd }}\right)$ has the following properties:

(i) It is homogeneous, i.e. for every $D \in \operatorname{Dense}(\mathbb{Q})$, Dense $(\mathbb{Q})\lceil D$ is isomorphic to Dense $(\mathbb{Q})$.

(ii) $(\operatorname{Dense}(\mathbb{Q}), \subseteq$ nwd $)$ is $\sigma$-closed.

(iii) $($ Dense $(\mathbb{Q}), \subseteq$ nwd $)$ satisfies $\mathfrak{c}^{+}$-c.c and fails $\mathfrak{c}-c c$.

Proof. (i) It follows from Sierpiński's characterization that $D$ is homeomorphic to $\mathbb{Q}$ for every $D \in \operatorname{Dense}(\mathbb{Q})$. Moreover, every nowhere dense subset of $D$ is nowhere dense in $\mathbb{Q}$.

For (ii) let $\left\langle D_{i}: i \in \omega\right\rangle$ be a sequence of elements of Dense $(\mathbb{Q})$ such that $D_{i+1} \subseteq_{\text {nwd }} D_{i}$ for every $i \in \omega$. Recursively pick $d_{n} \in U_{n} \cap \bigcap_{i \leq n} D_{i}$ and let $D=\left\{d_{i}: i \in \omega\right\}$. Then $D \in \operatorname{Dense}(\mathbb{Q})$ and $D \subseteq{ }^{*} D_{i}$ for every $i \in \omega$.

To prove (iii), for every $s \in 2^{<\omega}$ choose a set $D_{s} \in \operatorname{Dense}(\mathbb{Q})$ so that $D_{\emptyset}=\mathbb{Q}$ and $D_{s}$ is a disjoint union of $D_{s \wedge 0}$ and $D_{s \wedge 1}$. Then use (ii) to find for every $f \in 2^{\omega}$ a set $D_{f} \in \operatorname{Dense}(\mathbb{Q})$ such that $D_{f} \subseteq^{*} D_{f \nmid n}$ for every 
$n \in \omega$. Let $\mathcal{A}=\left\{D_{f}: f \in 2^{\omega}\right\}$. Obviously, a family of incompatible elements cannot have cardinality larger than $\mathfrak{c}=|\operatorname{Dense}(\mathbb{Q})|$.

Corollary 2.3. The partial order $\left(\operatorname{Dense}(\mathbb{Q}), \subseteq_{\text {nwd }}\right)$ is equivalent, as a forcing notion, to (Dense $\left.(X), \subseteq_{\text {nwd }}\right)$ for any separable metric space without isolated points.

Proof. Every separable metric space without isolated points has exactly $\mathfrak{c}$ countable dense sets. There is therefore a maximal antichain $\mathcal{A}$ in (Dense $\left.(X), \subseteq_{\text {nwd }}\right)$ of size $\mathfrak{c}$ consisting of countable dense subsets of $X$. The set $\mathcal{H}=\{C \in \operatorname{Dense}(X):(\exists D \in \mathcal{A})(C \subseteq D)\}$ is a dense set in the pre-order (Dense $(X), \subseteq_{\text {nwd }}$ ) and by Proposition 2.2 it is isomorphic to a dense subset of the pre-order $($ Dense $(\mathbb{Q}), \subseteq$ nwd $)$.

Given a partial (pre-)order $\mathbb{P}$ without minimal elements one can be interested in a "measure" of closedness of $\mathbb{P}$. The cardinal characteristic $\mathfrak{t}(\mathbb{P})$ is defined as the minimal $\kappa$ such that $\mathbb{P}$ is not $\kappa$-closed, i.e. there is a decreasing chain in $\mathbb{P}$ of size $\kappa$ without a lower bound in $\mathbb{P}$. A related cardinal invariant is $\mathfrak{p}(\mathbb{P})$ which denotes the minimal size of a downward directed subset of $\mathbb{P}$ without a lower bound in $\mathbb{P}$. The invariant $\mathfrak{t}(\mathbb{P})$ is always a regular infinite cardinal. For the partial order $\left([\omega]^{\omega}, \subseteq^{*}\right)$ these cardinal invariants are usually denoted simply by $\mathfrak{t}$ and $\mathfrak{p}$.

It is an open problem whether $\mathfrak{t}$ and $\mathfrak{p}$ can be consistently different. As far as we know there is even no known example of a $\sigma$-closed separative homogeneous partial order $\mathbb{P}$ with $\mathfrak{t}(\mathbb{P})$ and $\mathfrak{p}(\mathbb{P})$ distinct.

J. Cichoń $[\mathrm{Ci}]$ studied the (non-separative) partial order $\left(\operatorname{Dense}(\mathbb{Q}), \subseteq^{*}\right)$ and showed that $\mathfrak{t}=\mathfrak{t}\left(\operatorname{Dense}(\mathbb{Q}), \subseteq^{*}\right)$ and $\mathfrak{p}=\mathfrak{p}\left(\operatorname{Dense}(\mathbb{Q}), \subseteq^{*}\right)$. We will show that the same holds for the separative modification $\left(\operatorname{Dense}(\mathbb{Q}), \subseteq_{\text {nwd }}\right)$. We denote by $\mathfrak{t}_{\mathbb{Q}}$ the cardinal invariant $\mathfrak{t}\left(\operatorname{Dense}(\mathbb{Q}), \subseteq_{\text {nwd }}\right)$ and, similarly, $\mathfrak{p}_{\mathbb{Q}}=\mathfrak{p}\left(\operatorname{Dense}(\mathbb{Q}), \subseteq_{\text {nwd }}\right)$.

There is a natural embedding $\phi:[\omega]^{\omega} \rightarrow \operatorname{Dense}(\mathbb{Q})$, due to Sierpiński, defined by $\phi(X)=\left\{s \in 2^{<\omega}:|s| \in X\right\}$. This embedding preserves ordering and orthogonality but it is not a regular embedding since it does not preserve maximality of antichains. It will turn out useful nonetheless.

Theorem 2.4. $\mathfrak{t}=\mathfrak{t}_{\mathbb{Q}}$ and $\mathfrak{p}=\mathfrak{p}_{\mathbb{Q}}$.

Proof. The basic fact that $\mathfrak{p}_{\mathbb{Q}} \leq \mathfrak{t}_{\mathbb{Q}} \leq \operatorname{add}(\mathcal{M})$ follows directly from Theorem 3.2. We apologize for committing the crime of forward referencing.

First we prove that $\mathfrak{t}_{\mathbb{Q}} \leq \mathfrak{t}$. Fix $\kappa<\mathfrak{t}_{\mathbb{Q}}$ and let $\left\{T_{\alpha}: \alpha<\kappa\right\}$ be a $\subseteq^{*}-$ decreasing chain in $[\omega]^{\omega}$. Then $\left\{\phi\left(T_{\alpha}\right): \alpha<\kappa\right\}$ form a $\subseteq^{*}$-decreasing chain in Dense $(\mathbb{Q})$. As $\kappa<\mathfrak{t}_{\mathbb{Q}}$, there is a $D \in \operatorname{Dense}(\mathbb{Q})$ such that $D \subseteq$ nwd $\phi\left(T_{\alpha}\right)$ for all $\alpha<\kappa$. Treat the set $D$ as the rationals, and note that $\phi\left(T_{\alpha}\right) \cap D$ contains a dense open subset of $D$ for every $\alpha<\kappa$. As $\kappa<\mathfrak{t}_{\mathbb{Q}} \leq \operatorname{add}(\mathcal{M})$ an application of Theorem 1.4(ii) produces a dense set $D^{\prime} \subseteq D$ such that 
$D^{\prime} \subseteq^{*} \phi\left(T_{\alpha}\right)$. Put $T=\left\{|s|: s \in D^{\prime}\right\}$. Then $T$ is an infinite subset of $\omega$ and $T \subseteq^{*} T_{\alpha}$ for all $\alpha<\kappa$.

To prove that $\mathfrak{t} \leq \mathfrak{t}_{\mathbb{Q}}$ fix $\kappa<\mathfrak{t}$ and let $\left\{D_{\alpha}: \alpha<\kappa\right\}$ be a $\subseteq_{\text {nwd-decreasing }}$ chain in Dense $(\mathbb{Q})$. As, by Proposition $1.3($ ii) $), \kappa<\mathfrak{t} \leq \operatorname{add}(\mathcal{M})$, there is a dense $G_{\delta}$ set $Y \subseteq 2^{\omega}$ such that

(1) $Y \subseteq \widehat{D}_{\alpha}$ for all $\alpha<\kappa$,

(2) $Y \cap \widehat{N}_{\alpha, \beta}=\emptyset$, where $N_{\alpha, \beta}=D_{\alpha} \backslash D_{\beta}$, for all $\beta<\alpha<\kappa$.

For $f \in Y$ and $\alpha<\kappa$ let $A_{\alpha}^{f}=\left\{k \in \omega: f \nmid k \in D_{\alpha}\right\}$. Note that the set $A_{\alpha}^{f}$ is infinite for every $f \in Y$ and $\alpha<\kappa$. Also, for every $f$ in $Y$, the family $\left\{A_{\alpha}^{f}: \alpha<\kappa\right\}$ is a $\subseteq^{*}$-decreasing chain in $[\omega]^{\omega}$. As $\kappa<\mathfrak{t}$, there is a set $L_{f}$ which is a $\subseteq^{*}$-lower bound for $\left\{A_{\alpha}^{f}: \alpha<\kappa\right\}$. Let $C=\left\{c_{n}: n \in \omega\right\} \subseteq 2^{\omega}$ be a countable dense subset of $Y$ and put $E_{n}=\left\{c_{n}\left\lceil m: m \in L_{c_{n}}\right\}\right.$. For $\alpha<\kappa$ let

$$
g_{\alpha}(n)=\min \left\{k \in \omega:(\forall m>k)\left(c_{n}\left\lceil m \in E_{n} \Rightarrow c_{n}\left\lceil m \in D_{\alpha}\right)\right\} .\right.\right.
$$

As $\mathfrak{t} \leq \mathfrak{b}$ there is a function $g$ dominating all $g_{\alpha}, \alpha<\kappa$. Put

$$
D=\bigcup_{n \in \omega}\left\{c_{n}\left\lceil m \in E_{n}: m \geq g(n)\right\} .\right.
$$

Since $C$ is a dense subset of $2^{\omega}, D$ is in Dense $(\mathbb{Q})$. It is clear that $D \subseteq^{*} D_{\alpha}$ for every $\alpha<\kappa$. So, we have proved that $\kappa<\mathfrak{t}_{\mathbb{Q}}$.

The proof that $\mathfrak{p}=\mathfrak{p}_{\mathbb{Q}}$ is analogous, only simpler.

Another cardinal invariant studied by J. Cichon in $[\mathrm{Ci}]$ is defined as follows: Given a family $\mathcal{S} \subseteq[\omega]^{\omega}$ we say that a function $\psi: \omega \rightarrow \mathbb{Q}$ is a dense embedding of $\mathcal{S}$ if $\psi$ is one-to-one and $\psi[S] \in \operatorname{Dense}(\mathbb{Q})$ for every $S \in \mathcal{S}$. The cardinal invariant deq denotes the minimal cardinality of a family $\mathcal{S} \subseteq[\omega]^{\omega}$ for which there is no dense embedding. Recall that the reaping number $\mathfrak{r}$ denotes the minimal size of a family $\mathcal{R} \subseteq[\omega]^{\omega}$ such that for every $A \in[\omega]^{\omega}$ there is an $R \in \mathcal{R}$ such that $R \subseteq^{*} A$ or $R \cap A$ is finite. Cichon showed that deq $\leq \mathfrak{r}$ and found some lower bounds for deq. The next proposition shows that the two numbers actually coincide. Let $A, B$ be infinite subsets of $\omega$. We say that $A$ splits $B$ if both $A \cap B$ and $B \backslash A$ are infinite.

Proposition 2.5. deq $=\mathfrak{r}$.

Proof. Let $\mathcal{R} \subseteq[\omega]^{\omega}$ be a reaping family of size $\mathfrak{r}$. Aiming for contradiction suppose that $\psi$ is a dense embedding of $\mathcal{R}$. Split $\mathbb{Q}$ into two disjoint open sets $U, V$. Let $A=\psi^{-1}[U]$. It is easily seen that $A$ splits every element of $\mathcal{R}$, which contradicts the assumption that $\mathcal{R}$ was a reaping family.

For the other direction, let $\mathcal{S} \subseteq[\omega]^{\omega}$ be of size strictly less than $\mathfrak{r}$. We need to show that there is a dense embedding of $\mathcal{S}$. Rather then constructing 
an embedding we show that there is a topology on $\omega$ homeomorphic to $\mathbb{Q}$ in which all sets in $\mathcal{S}$ are dense. Recursively construct sets $I_{n}, n \in \omega$, so that

(1) $I_{0}$ splits all elements of $\mathcal{S}$,

(2) $I_{n+1}$ splits all infinite intersections of finitely many elements of the family $\mathcal{S} \cup\left\{I_{m}: m \leq n\right\} \cup\left\{\omega \backslash I_{m}: m \leq n\right\}$,

(3) for every pair $i \neq j \in \omega$ there is an $n \in \omega$ such that $\left|I_{n} \cap\{i, j\}\right|=1$, i.e. $I_{n}$ separates $i$ and $j$.

To do this is easy: existence of $I_{n+1}$ follows directly from the fact that the family of all intersections of finitely many elements of the family $\mathcal{S} \cup\left\{I_{m}\right.$ : $m \leq n\}$ has size strictly less than $\mathfrak{r}$. Note that declaring the family $\left\{I_{n}\right.$ : $n \in \omega\}$ to be a clopen subbase defines a Hausdorff, regular, zero-dimensional second countable topology on $\omega$ in which all elements of $\mathcal{S}$ are dense.

A family $\mathcal{I} \subseteq[\omega]^{\omega}$ is independent provided that $\bigcap \mathcal{F} \backslash \cup \mathcal{G} \neq \emptyset$ for any non-empty and disjoint $\mathcal{F}, \mathcal{G} \in[\mathcal{I}]^{<\omega}$. Similarly a family $\mathcal{I} \subseteq \operatorname{Dense}(\mathbb{Q})$ is said to be dense-independent if $\bigcap \mathcal{F} \backslash \bigcup \mathcal{G} \in \operatorname{Dense}(\mathbb{Q})$ for any disjoint finite subsets $\mathcal{F}, \mathcal{G}$ of $\mathcal{I}$. The cardinal invariant $\mathfrak{i}$ denotes the minimal cardinality of a maximal independent family. Similarly, $\mathfrak{i}_{\mathbb{Q}}$ denotes the minimal cardinality of a maximal dense-independent family. Next we show that these two cardinal numbers actually coincide.

Proposition 2.6. $\mathfrak{i}=\mathfrak{i}_{\mathbb{Q}}$.

Proof. To prove that $\mathfrak{i} \leq \mathfrak{i}_{\mathbb{Q}}$, identify $\mathbb{Q}$ with the set $\left\{q \in 2^{\omega}:\left(\forall^{\infty} i \in \omega\right)\right.$ $(q(i)=0)\}$ with the topology inherited from $2^{\omega}$. Let $\mathcal{I}$ be a maximal denseindependent family of size $\mathfrak{i}_{\mathbb{Q}}$. For $n \in \omega$ let $I_{n}=\{q \in \mathbb{Q}: q(n)=1\}$. Note that the sets $I_{n}$ and their complements form a subbase for the topology on $\mathbb{Q}$. Moreover, the family $\mathcal{I}^{\prime}=\mathcal{I} \cup\left\{I_{n}: n \in \omega\right\}$ is an independent family of subsets of $\mathbb{Q}$. We will show that it is in fact maximal. Aiming for a contradiction suppose that $I$ is a subset of $\mathbb{Q}$ such that $\mathcal{I}^{\prime} \cup\{I\}$ is independent. Then $I \in \operatorname{Dense}(\mathbb{Q})$ because $\{I\} \cup\left\{I_{n}: n \in \omega\right\}$ is an independent family and the Boolean combinations of the elements of $\left\{I_{n}: n \in \omega\right\}$ form a base for the topology on $\mathbb{Q}$. Similar reasoning shows that the family $\mathcal{I} \cup\{I\}$ is then dense-independent, contradicting the maximality of $\mathcal{I}$. As $|\mathcal{I}|=\left|\mathcal{I}^{\prime}\right|$ we are done.

For the other direction let $\mathcal{I}$ be a maximal independent family. Let $\left\{J_{n}\right.$ : $n \in \omega\}$ be a sequence of distinct elements of $\mathcal{I}$. By changing them by a finite set if necessary, we can assume that they separate points, i.e. for every pair $i \neq j \in \omega$ there is an $n \in \omega$ such that $\left|J_{n} \cap\{i, j\}\right|=1$. The sets $\left\{J_{n}: n \in \omega\right\}$ then form a clopen subbase of a Hausdorff, regular, zero-dimensional second countable topology on $\omega$ in which all elements of $\mathcal{I} \backslash\left\{J_{n}: n \in \omega\right\}$ are dense. Identify $\mathbb{Q}$ with $\omega$ equipped with this topology. It is again routine to verify that the family $\mathcal{I} \backslash\left\{J_{n}: n \in \omega\right\}$ is a maximal dense-independent family. 
3. Dense $(\mathbb{Q})$ as a forcing notion: $\mathcal{P}(\omega) /$ fin and Dense $(\mathbb{Q})$ are not quite the same. A basic fact about $\mathcal{P}(\omega) /$ fin is that the partial order $\left([\omega]^{\omega}, \subseteq^{*}\right)$ is not isomorphic to its product $\left([\omega]^{\omega}, \subseteq^{*}\right) \times\left([\omega]^{\omega}, \subseteq^{*}\right)$, as $\beta \omega \backslash \omega$ is not homeomorphic to $\beta \omega \backslash \omega \times \beta \omega \backslash \omega$. The situation is quite different for $\operatorname{Dense}(\mathbb{Q})$.

Proposition 3.1. The partial order $\left(\operatorname{Dense}(\mathbb{Q}), \subseteq_{\mathrm{nwd}}\right)$ is isomorphic to $\left(\operatorname{Dense}(\mathbb{Q}), \subseteq_{\text {nwd }}\right)^{\omega}$.

Proof. Partition $\mathbb{Q}$ into pairwise disjoint non-empty open sets $V_{n}, n \in \omega$. Note that $\left(\operatorname{Dense}\left(V_{n}\right), \subseteq_{\text {nwd }}\right)$ is isomorphic to $\left(\operatorname{Dense}(\mathbb{Q}), \subseteq_{\text {nwd }}\right)$. Define

$$
\Psi: \operatorname{Dense}(\mathbb{Q}) \rightarrow \prod\left\{\operatorname{Dense}\left(V_{n}\right): n \in \omega\right\}
$$

by $\Psi(D)=\left\langle D \cap V_{n}: n \in \omega\right\rangle$. It is easily seen that this map induces the desired isomorphism.

The distributivity number of a separative partial pre-order $\mathbb{P}$ without minimal elements, denoted by $\mathfrak{h}(\mathbb{P})$, is defined as the minimal size of a family of maximal antichains in $\mathbb{P}$ without common refinement. For $\mathbb{P}$ homogeneous, $\mathfrak{h}(\mathbb{P})$ is equal to the minimal size of a collection of dense downward closed subsets of $\mathbb{P}$ whose intersection is empty. The number $\mathfrak{h}(\mathbb{P})$ is equal to $\mathfrak{h}(\mathbb{B})$, where $\mathbb{B}$ is the complete Boolean algebra determined by $\mathbb{P}$. Note that $\mathfrak{t}(\mathbb{P}) \leq$ $\mathfrak{h}(\mathbb{P})$ for every separative partial pre-order $\mathbb{P}$. Again, $\mathfrak{h}\left([\omega]^{\omega}, \subseteq^{*}\right)$ is denoted simply by $\mathfrak{h}$ and we denote $\mathfrak{h}(\operatorname{Dense}(\mathbb{Q}), \subseteq$ nwd $)$ by $\mathfrak{h}_{\mathbb{Q}}$. It was shown in $[\mathrm{SS}]$ that the distributivity number of the free product $\mathcal{P}(\omega) /$ fin $\otimes \mathcal{P}(\omega) /$ fin is consistently strictly smaller than $\mathfrak{h}$. By Proposition 3.1 this does not happen in the case of Dense $(\mathbb{Q})$.

The following theorem shows that unlike in the case of $\mathcal{P}(\omega)$ /fin the properties of the partial order Dense $(\mathbb{Q})$ depend on the topological properties of the real line.

Theorem 3.2. $\mathfrak{h}_{\mathbb{Q}} \leq \operatorname{add}(\mathcal{M})$.

Proof. Let $\left\{V_{n}: n \in \omega\right\}$ be an enumeration of all non-empty clopen subsets of the Cantor set $2^{\omega}$. For each $n \in \omega$ fix a family $\left\{K_{\alpha}^{n}: \alpha<\operatorname{add}(\mathcal{M})\right\}$ of meager subsets of $V_{n}$ such that $\bigcup\left\{K_{\alpha}^{n}: \alpha<\operatorname{add}(\mathcal{M})\right\}$ is not meager in $V_{n}$. Put $M_{\alpha}=\bigcup\left\{K_{\beta}^{n}: \beta<\alpha\right.$ and $\left.n \in \omega\right\}$. Note that each $M_{\alpha}$ is meager in $2^{\omega}$ and that they form an increasing chain. For $\alpha<\operatorname{add}(\mathcal{M})$ put

$$
\mathcal{H}_{\alpha}=\left\{D \in \operatorname{Dense}(\mathbb{Q}): \widehat{D} \cap M_{\alpha} \text { is nowhere dense in } 2^{\omega}\right\} .
$$

Note that:

(a) $\mathcal{H}_{\alpha}$ is downward closed in $(\operatorname{Dense}(\mathbb{Q}), \subseteq$ nwd $)$, i.e. if $D \in \mathcal{H}_{\alpha}$ and $C \subseteq_{\text {nwd }} D$ then $C \in \mathcal{H}_{\alpha}$.

This follows directly from Fact 1.2. 
(b) $\mathcal{H}_{\alpha}$ is dense in $\operatorname{Dense}(\mathbb{Q})$.

To see this, let $D \in \operatorname{Dense}(\mathbb{Q})$. As $M_{\alpha}$ is meager, there is a dense $G_{\delta}$ set $Y \subseteq \widehat{D}$ disjoint from $M_{\alpha}$. Write $Y$ as the intersection of a decreasing sequence of $W_{n}, n \in \omega$, where each $W_{n}$ is open dense in $2^{\omega}$. For every $n \in \omega$ let $A_{n}$ be a maximal antichain in $D$ such that $\langle s\rangle \subseteq W_{n}$ for every $s \in A_{n}$ and $A_{n+1}$ refines $A_{n}$. Note that $\bigcup\left\{\langle s\rangle: s \in A_{n}\right\} \subseteq W_{n}$. Let $C=\bigcup\left\{A_{n}: n \in \omega\right\}$. Obviously, $C \subseteq D$ is dense. It is also easy to see that $\widehat{C} \subseteq Y$.

To finish the proof it suffices to check that:

(c) $\bigcap\left\{\mathcal{H}_{\alpha}: \alpha<\operatorname{add}(\mathcal{M})\right\}=\emptyset$.

To that end take $D \in \operatorname{Dense}(\mathbb{Q})$. As $\bigcup\left\{K_{\alpha}^{n}: \alpha<\operatorname{add}(\mathcal{M})\right\}$ is not meager in $V_{n}$ and $\widehat{D}$ is dense $G_{\delta}$ in $2^{\omega}$, for every $n \in \omega$ there is an $\alpha_{n}<\operatorname{add}(\mathcal{M})$ such that $\widehat{D} \cap V_{n} \cap K_{\alpha_{n}}^{n} \neq \emptyset$. Pick $c_{n} \in \widehat{D} \cap V_{n} \cap K_{\alpha_{n}}^{n}$. The set $\left\{c_{n}: n \in \omega\right\}$ is dense in $2^{\omega}$. As $\operatorname{add}(\mathcal{M})$ is a regular uncountable cardinal, there is an $\alpha<\operatorname{add}(\mathcal{M})$ such that $\alpha_{n}<\alpha$ for every $n \in \omega$. Then, however, $\left\{c_{n}: n \in \omega\right\} \subseteq M_{\alpha} \cap \widehat{D}$, which means that $D \notin \mathcal{H}_{\alpha}$.

Let us remark that the equality in 3.2 is not provable (see 3.5(i)).

Theorem 3.3 (Base tree). There is a family $T \subseteq \operatorname{Dense}(\mathbb{Q})$ such that

(1) $T$ is a tree, ordered by $\supset^{*}$, of height $\mathfrak{h}_{\mathbb{Q}}$,

(2) each level of $T$ is a maximal antichain in $\operatorname{Dense}(\mathbb{Q})$,

(3) each $D \in T$ has $\mathfrak{c}$ immediate successors,

(4) for every $D \in \operatorname{Dense}(\mathbb{Q})$ there is a $C \in T$ such that $C \subseteq D$.

Proof. It follows directly from Theorem 1.13 of [BS] that there is a family $T^{\prime} \subseteq \operatorname{Dense}(\mathbb{Q})$ such that

(1) $T^{\prime}$ is a dense subset of $\operatorname{Dense}(\mathbb{Q})$,

(2) $T^{\prime}$ is a tree (ordered by $\supset_{\text {nwd }}$ ) of height $\mathfrak{h}_{\mathbb{Q}}$,

(3) each level of $T^{\prime}$ is a maximal antichain in $\operatorname{Dense}(\mathbb{Q})$,

(4) each $D \in T^{\prime}$ has $\mathfrak{c}$ immediate successors.

Enumerate all nowhere dense subsets of $\mathbb{Q}$ as $\left\{M_{\xi}: \xi<\mathfrak{c}\right\}$. We will construct a tree $T$ by induction on levels. For $\alpha=\beta+1$ let $\mathcal{A}$ be a maximal antichain in Dense $(\mathbb{Q})$ refining both $T_{\beta}$ and $T_{\alpha}^{\prime}$ such that for every $D \in T_{\beta}$ there are $\mathfrak{c}$ elements of $\mathcal{A}$ below $D$. For every $D \in T_{\beta}$ enumerate all immediate successors of $D($ in $\mathcal{A})$ as $\left\{C_{\xi}^{D}: \xi<\mathfrak{c}\right\}$ and let $T_{\alpha}=\left\{C_{\xi}^{D} \backslash M_{\xi}: D \in T_{\beta}\right.$ and $\xi<\mathfrak{c}$.

For $\alpha<\mathfrak{h}_{\mathbb{Q}}$ limit, let $\mathcal{A}$ be a maximal antichain in Dense( $\left.\mathbb{Q}\right)$ refining all $T_{\beta}, \beta<\alpha$, and let $\mathcal{H}$ be the downward closure of $\mathcal{A}$. For every $D \in \mathcal{H}$ and every $\beta<\alpha$ there is a unique $C_{\beta} \in T_{\beta}$ such that $N_{\beta}=D \backslash C_{\beta}$ is nowhere dense in $D$. As $D$ is homeomorphic to $\mathbb{Q}$ and as $\alpha<\mathfrak{h}_{\mathbb{Q}} \leq \operatorname{add}(\mathcal{M})$, by Theorem 1.4, there is a dense subset $C_{D} \subseteq D$ such that $C_{D} \cap N_{\beta}$ is finite, 
or equivalently, $C_{D} \subseteq^{*} C_{\beta}$ for every $\beta<\alpha$. Let $T_{\alpha}$ be a maximal antichain contained in $\left\{C_{D}: D \in \mathcal{H}\right\}$.

The tree $T=\bigcup\left\{T_{\alpha}: \alpha<\mathfrak{h}_{\mathbb{Q}}\right\}$ is dense as, by construction, $T_{\alpha+1}$ refines $T_{\alpha}^{\prime}$ and $T$ is ordered by $\supseteq *$. To see that for every $D \in \operatorname{Dense}(\mathbb{Q})$ there is a $C \in T$ such that $C \subseteq D$, fix $D$. There is an $E$ in $T$ such that $E \subset$ nwd $D$. There is a $\xi<\mathfrak{c}$ such that $M_{\xi}=E \backslash D$. Then $C=C_{\xi}^{E} \subseteq D$.

Cichoń $[\mathrm{Ci}]$ also investigated a cardinal invariant $\mathfrak{s}_{\mathbb{Q}}$ which is defined as the minimal size of a family $\mathcal{S} \subseteq$ Dense $(\mathbb{Q})$ such that for every $D \in \operatorname{Dense}(\mathbb{Q})$ there is an $S \in \mathcal{S}$ such that both $S \cap D$ and $D \backslash S$ are dense. He showed that $\mathfrak{s}_{\mathbb{Q}}$ is less than or equal to the cardinal invariant $\aleph_{0}-\mathfrak{s}$ introduced by Malykhin and studied in $[\mathrm{KW}]$. The cardinal invariant $\aleph_{0}-\mathfrak{s}$ is defined as the minimal cardinality of a family $\mathcal{S} \subseteq[\omega]^{\omega}$ such that for any countable $\mathcal{B} \subseteq[\omega]^{\omega}$ there is a set $S \in \mathcal{S}$ which splits all elements of $\mathcal{B}$. Obviously, $\mathfrak{s} \leq \aleph_{0}-\mathfrak{s}$ and also $\aleph_{0}-\mathfrak{s} \leq \mathfrak{d}$. It is an open problem whether $\mathfrak{s}<\aleph_{0}-\mathfrak{s}$ is consistent.

Proposition 3.4. $\mathfrak{h}_{\mathbb{Q}} \leq \mathfrak{s}_{\mathbb{Q}}$.

Proof. The proof is quite analogous to the proof that $\mathfrak{h} \leq \mathfrak{s}$. Fix a family $\mathcal{S} \subseteq \operatorname{Dense}(\mathbb{Q})$ such that for every $D \in \operatorname{Dense}(\mathbb{Q})$ there is an $S \in \mathcal{S}$ such that both $S \cap D$ and $D \backslash S$ are dense. For every $S \in \mathcal{S}$ let

$$
\mathcal{H}_{S}=\{D \in \operatorname{Dense}(\mathbb{Q}): D \cap S \in \operatorname{nwd} \text { or } D \backslash S \in \operatorname{nwd}\} .
$$

This works.

THEOREM 3.5. The following are all relatively consistent with ZFC:

(i) $\mathfrak{h}_{\mathbb{Q}}<\operatorname{add}(\mathcal{M})$,

(ii) $\mathfrak{h}_{\mathbb{Q}}<\mathfrak{h}$,

(iii) $\mathfrak{t}<\mathfrak{h}_{\mathbb{Q}}$.

Proof. (i) holds in the Hechler model, i.e. the model obtained from a model of $\mathrm{CH}$ by forcing with finite support iteration of length $\omega_{2}$ of Hechler forcing. As Hechler forcing adds both a Cohen real and a dominating real, $\operatorname{add}(\mathcal{M})=\omega_{2}$ in the Hechler model. On the other hand, Hechler forcing preserves splitting families; hence $\mathfrak{s}=\omega_{1}$ in the Hechler model (see e.g. $[\mathrm{BJ}])$. In $[\mathrm{KW}]$ it is shown that if $\mathfrak{s}<\operatorname{cov}(\mathcal{M})$ then $\aleph_{0}-\mathfrak{s}=\mathfrak{s}$. Using Cichon's result we conclude that $\mathfrak{s}_{\mathbb{Q}}=\omega_{1}$ and by Proposition $3.4, \mathfrak{h}_{\mathbb{Q}}=\omega_{1}$.

(ii) holds in the iterated Mathias model. It was probably first observed in $\left[\right.$ Do] that $\mathfrak{h}=\mathfrak{c}=\omega_{2}$ in the Mathias model. The fact that $\operatorname{add}(\mathcal{M})=\omega_{1}$ in the Mathias model (see e.g. [BJ]) follows from the fact that Mathias forcing has the Laver property and hence in the Mathias model there are no reals Cohen generic over the ground model. By Theorem 3.2, the result follows.

(iii) holds for example in a model obtained from a model of $\mathrm{CH}$ by forcing with the free product $\mathbb{B} \otimes \operatorname{Fn}\left(\omega_{1}, 2, \omega_{1}\right)$, where $\mathbb{B}$ denotes the Solovay- 
Tennenbaum algebra for forcing Martin's Axiom and failure of CH. The argument is similar to the one in [BPS]).

The reaping number $\mathfrak{r}$ was mentioned before Proposition 2.5. Next we deal with its analogue in the context of Dense $(\mathbb{Q})$. Call a family $\mathcal{R} \subseteq$ Dense $(\mathbb{Q})$ dense-reaping if no dense set $D$ splits all elements of $\mathcal{R}$ into dense pieces, i.e. for every $D \in \operatorname{Dense}(\mathbb{Q})$ there is an $R \in \mathcal{R}$ such that $R \backslash D \notin$ Dense $(\mathbb{Q})$ or $R \cap D \notin \operatorname{Dense}(\mathbb{Q})$, or equivalently, for every $D \in \operatorname{Dense}(\mathbb{Q})$ there are $R \in \mathcal{R}$ and $U \subseteq \mathbb{Q}$ open such that $D \cap R \cap U=\emptyset$ or $R \cap U \subseteq D$. The cardinal invariant $\mathfrak{r}_{\mathbb{Q}}$ denotes the minimal size of a dense-reaping family. In [MHD] it is shown that $\diamond\left(\mathfrak{r}_{\mathbb{Q}}\right)$ implies $\mathfrak{i}=\omega_{1}$.

Theorem 3.6. $\max \{\mathfrak{r}, \operatorname{cof}(\mathcal{M})\} \leq \mathfrak{r}_{\mathbb{Q}} \leq \mathfrak{i}$.

Proof. The fact that $\mathfrak{r}_{\mathbb{Q}} \leq \mathfrak{i}$ follows directly from Proposition 2.6 and the simple observation that $\mathfrak{r}_{\mathbb{Q}} \leq \mathfrak{i}_{\mathbb{Q}}$.

To see that $\mathfrak{r} \leq \mathfrak{r}_{\mathbb{Q}}$ fix $\kappa<\mathfrak{r}$ and a family $\mathcal{R}=\left\{D_{\alpha}: \alpha<\kappa\right\} \subseteq$ Dense $(\mathbb{Q})$. Consider $\mathcal{R}^{\prime}=\left\{D_{\alpha} \cap U_{n}: \alpha<\kappa\right.$ and $\left.n \in \omega\right\}$. It has also size $\kappa$ and as $\kappa<\mathfrak{r}$ it is not a reaping family; hence there is a set $D \subseteq \mathbb{Q}$ which splits all elements of $\mathcal{R}^{\prime}$ into infinite pieces. It follows that $D$ is dense and splits each $D_{\alpha}$ into two dense sets and hence $\kappa<\mathfrak{r}_{\mathbb{Q}}$.

We will use Theorem 1.6 and prove that $\kappa<\mu_{\mathbb{Q}} \Rightarrow \kappa<\mathfrak{r}_{\mathbb{Q}}$. To that end, assume $\kappa<\mu_{\mathbb{Q}}$ and consider a base $\left\{U_{n}: n \in \omega\right\}$ for the topology of $\mathbb{Q}$ and a family $\mathcal{R}=\left\{R_{\alpha}: \alpha<\kappa\right\}$ of dense subsets of $\mathbb{Q}$. Build sequences of disjoint nwd subsets $F_{n}^{0}, F_{n}^{1} \subseteq \mathbb{Q}, n \in \omega$, such that

(1) $(\forall n \in \omega)\left(F_{n}^{0} \cup F_{n}^{1} \subseteq U_{n}\right)$,

(2) $(\forall m, n \in \omega)(\forall i, j \in\{0,1\})\left(m \neq n \Rightarrow F_{m}^{i} \cap F_{n}^{j}=\emptyset\right)$.

By induction, assume $F_{k}^{i}$ have been chosen for $i \in\{0,1\}$ and $k<n$. Then $F=\bigcup_{k<n}\left(F_{k}^{0} \cup F_{k}^{1}\right)$ is nowhere dense and hence $U_{n} \backslash F$ is non-empty; furthermore, it is homeomorphic to $\mathbb{Q}$. Thus, since $\kappa<\mu_{\mathbb{Q}}$, there is some $F_{n}^{0} \subseteq U_{n} \backslash F$ such that $F_{n}^{0} \cap R_{\alpha} \neq \emptyset$ for every $\alpha<\kappa$, and repeating the same argument find $F_{n}^{1} \subseteq U_{n} \backslash\left(F \cup F_{n}^{0}\right)$ such that also $F_{n}^{1} \cap R_{\alpha} \neq \emptyset$ for every $\alpha<\kappa$. This completes the construction of the sequences.

Now let $D=\bigcup_{n \in \omega} F_{n}^{0}$. By construction $D$ is dense and $D$ does not get reaped by the family $\mathcal{R}$. Therefore $\kappa<\mathfrak{r}_{\mathbb{Q}}$.

Note that it follows that $\mathfrak{s} \mathbb{Q} \leq \mathfrak{r}_{\mathbb{Q}}$ while $\mathfrak{r}$ and $\mathfrak{s}$ are not provably comparable.

TheOREM 3.7. It is relatively consistent with $Z F C$ that $\mathfrak{r}<\mathfrak{r}_{\mathbb{Q}}$.

Proof. By Theorem 3.6 this holds in any model where $\mathfrak{r}<\mathfrak{d}$; in particular, it holds in the iterated Miller model (see [Mi] or $[\mathrm{Bl}]$ ).

It is worth noting that Theorem 3.6 gives $\operatorname{cof}(\mathcal{M}) \leq \mathfrak{i}$, which improves the best known result placing $\mathfrak{i}$ among other cardinal invariants: Shelah's 
result that $\mathfrak{d} \leq \mathfrak{i}$ (see $[\mathrm{Va}]$ ). The analogous claim for measure does not hold.

THEOREM 3.8. It is consistent with $Z F C$ that $\mathfrak{i}<\operatorname{non}(\mathcal{N})$.

Proof. Start with a model of $\operatorname{non}(\mathcal{N})>\omega_{1}$. It is shown in [BJ, Lemma 6.5.27 and Theorem 6.5.31] that a finite support iteration of $\sigma$-centred forcings does not decrease $\operatorname{non}(\mathcal{N})$, thus all we need to do is find a forcing iteration of $\sigma$-centred forcings which forces $\mathfrak{i}$ to be small.

If $\mathcal{I}$ is a countable independent family, find a maximal filter $\mathcal{F}$ with the property

$$
(\forall F \in \mathcal{F})\left(\forall H \in[\mathcal{I}]^{<\aleph_{0}}\right)(\forall \xi: H \rightarrow 2)\left(\left|F \cap I_{\xi}\right|=\aleph_{0}\right),
$$

where $I_{\xi}=\bigcap\{I: \xi(I)=1\} \cap \bigcap\{\omega \backslash I: \xi(I)=0\}$. Let

$$
\mathbb{M}_{\mathcal{F}}=\left\{\langle s, F\rangle: s \in[\omega]^{<\aleph_{0}} \wedge F \in \mathcal{F}\right\}
$$

with the ordering $\langle s, F\rangle \leq\left\langle t, F^{\prime}\right\rangle$ if and only if $s \subseteq t \cup F^{\prime} \wedge F \subseteq F^{\prime}$. This forcing is sometimes called the Mathias forcing with respect to $\mathcal{F}$. The $\mathbb{M}_{\mathcal{F}}$-generic real $I$ will be a subset of $\omega$ which can be added to the countable family $\mathcal{I}$ without affecting its independence. It is also clear that $\mathbb{M}_{\mathcal{F}}$ is a $\sigma$-centred forcing.

Consequently, all we need is to iterate the forcings $\mathbb{M}_{\mathcal{F}}$ to produce a maximal independent family on $\omega$. So, let $\mathbb{P}=\left\langle\mathbb{P}_{\alpha} ; \dot{\mathbb{Q}}_{\alpha}: \alpha<\omega_{1}\right\rangle$ be the finite support iteration inductively defined as follows: Assuming $\mathbb{P}_{\alpha}$ is defined and that $\dot{\mathcal{I}}_{\alpha}$ is a $\mathbb{P}_{\alpha}$-name for a countable independent family on $\omega$, let $G_{\alpha}$ any $\mathbb{P}_{\alpha}$-generic filter over $V$. In $V\left[G_{\alpha}\right]$, take a maximal filter $\mathcal{F}$ satisfying the above mentioned property with $\mathcal{I}_{\alpha}$ in place of $\mathcal{I}$. Then let $\mathbb{Q}_{\alpha}$ be the forcing $\mathbb{M}_{\mathcal{F}}$, as defined in $V\left[G_{\alpha}\right]$. Thus $\mathbb{P}_{\alpha+1}$ is defined; $\dot{\mathcal{I}}_{\alpha+1}$ will be a $\mathbb{P}_{\alpha+1}$-name for $\mathcal{I}_{\alpha}$ augmented by the generic subset of $\omega$ coded by the $\mathbb{Q}_{\alpha}$-generic filter over $V\left[G_{\alpha}\right]$, and for $\alpha$ limit, $\mathcal{I}_{\alpha}=\bigcup_{\beta<\alpha} \mathcal{I}_{\beta}$.

Let $G$ be $\mathbb{P}$-generic over the ground model, $V$, and let $I_{\alpha}$ be the $\alpha$ th generic real added with this iteration. A simple reflection argument in $V[G]$ shows that $\mathcal{I}=\left\{I_{\alpha}: \alpha<\omega_{1}\right\}$ is a maximal independent family. Thus $V[G] \models \mathfrak{i}=\aleph_{1}$.

The cardinal invariant $\mathfrak{r}_{\mathbb{Q}}$ is a Borel invariant dual to $\mathfrak{s}_{\mathbb{Q}}$. However, from the point of view of the partial order Dense $(\mathbb{Q})$, a more natural notion is the following: Call a family $\mathcal{R} \subseteq \operatorname{Dense}(\mathbb{Q})$ strongly dense-reaping if for every $D \in \operatorname{Dense}(\mathbb{Q})$ there is an $R \in \mathcal{R}$ such that $R \backslash D$ is nowhere dense or $R \cap D$ is nowhere dense.

THEOREM 3.9. Every strongly dense-reaping family has size $\mathfrak{c}$.

Proof. Let $\mathcal{R} \subseteq$ Dense $(\mathbb{Q})$ be of size less than $\mathfrak{c}$. We will show that $\mathcal{R}$ is not strongly dense-reaping. To that end split $\mathbb{Q}$ into two open sets $U, V$, let $\left\{A_{\alpha}: \alpha<\mathfrak{c}\right\}$ be an almost disjoint family of dense subsets of $U$ and let $\left\{B_{\alpha}: \alpha<\mathfrak{c}\right\}$ be an almost disjoint family of dense subsets of $V$. As $|\mathcal{R}|<\mathfrak{c}$, 
there are $\alpha, \beta<\mathfrak{c}$ such that $R \cap U \nsubseteq_{\text {nwd }} A_{\alpha}$ and $R \cap V \nsubseteq_{\text {nwd }} B_{\alpha}$ for every $R \in \mathcal{R}$. Set $D=A_{\alpha} \cup\left(V \backslash B_{\beta}\right)$. This is clearly a dense subset of $\mathbb{Q}$ which is not strongly reaped by any element of $\mathcal{R}$.

Next, we mention some elementary properties of the generic extension obtained by forcing with $\operatorname{Dense}(\mathbb{Q})$. Let $U$ be a Dense $(\mathbb{Q})$-generic filter over $V$. In $V[U], \mathfrak{c}=\mathfrak{h}_{\mathbb{Q}}^{V}$ and $\mathcal{P}(\kappa)=\mathcal{P}(\kappa)^{V}$ for all $\kappa<\mathfrak{h}_{\mathbb{Q}}$; in particular Dense $(\mathbb{Q})$ does not add any new subsets of $\mathbb{Q}$. This follows from homogeneity of Dense $(\mathbb{Q})$ and standard reformulation of distributivity. Just as $\mathcal{P}(\omega) /$ fin generically adds a selective ultrafilter on $\omega$, the forcing Dense $(\mathbb{Q})$ adds a free filter on $\mathbb{Q}$. Recall that a free filter on a countable set is a simple $P_{\kappa}$-filter if it is generated by a $\subseteq^{*}$-decreasing chain of length $\kappa$. A filter $\mathcal{F}$ on $\omega$ is a $Q$-filter if for every partition $\left\langle I_{n}: n \in \omega\right\rangle$ of $\omega$ into finite sets there is an $X \in \mathcal{F}$ such that $\left|X \cap I_{n}\right| \leq 1$ for every $n \in \omega$.

Proposition 3.10. In $V[U], U$ is a simple $P_{\mathfrak{c}}$-filter on $\mathbb{Q}$ which is also a $Q$-filter. In particular, it is non-measurable and does not have the Baire property and has the following selection property: Given a partition $\left\langle I_{n}\right.$ : $n \in \omega\rangle$ of $\mathbb{Q}$ into nowhere dense sets there is an $X \in U$ such that $\left|X \cap I_{n}\right| \leq 1$ for every $n \in \omega$.

Proof. The generic filter $U$ introduces a branch through the base tree constructed in Theorem 3.3. Condition (1) of the theorem guarantees that the branch forms a $\subseteq^{*}$-decreasing chain of length $\mathfrak{h}_{\mathbb{Q}}$. As no new subsets of $\mathbb{Q}$ are added, genericity of $U$ and condition (4) of the theorem ensure that this branch forms a base of $U$. As $\mathfrak{h}_{\mathbb{Q}}^{V}=\mathfrak{c}$ in $V[U]$, the proof that $U$ is a simple $P_{\mathfrak{c}}$-filter is finished.

Next we prove that $U$ has the selection property and in particular is a $Q$-filter. As Dense $(\mathbb{Q})$ does not add any new reals, all that needs to be shown is that given a partition $\left\langle I_{n}: n \in \omega\right\rangle$ of $\mathbb{Q}$ into nowhere dense sets, the set $\mathcal{D}=\left\{D \in \operatorname{Dense}(\mathbb{Q}):(\forall n \in \omega)\left(\left|D \cap I_{n}\right| \leq 1\right)\right\}$ is dense in the partial order Dense $(\mathbb{Q})$. To see this fix $C \in \operatorname{Dense}(\mathbb{Q})$ and recursively choose $q_{n} \in U_{n} \cap C$ so that no two distinct $q_{n}$ are in the same part of the partition. It is easily verified that $D=\left\{q_{n}: n \in \omega\right\} \subseteq C$ is an element of $\mathcal{D}$.

We conclude this section with the list of problems which are still open.

QUESTIONS 3.11. Are the following relatively consistent with ZFC?

(1) $\mathfrak{h}<\mathfrak{h}_{\mathbb{Q}}$

$(2) \mathfrak{s}<\mathfrak{s}_{\mathbb{Q}}$,

(3) $\mathfrak{s} \mathbb{Q}<\mathfrak{s}$,

(4) $\mathfrak{h}_{\mathbb{Q}}<\mathfrak{s} \mathbb{Q}$,

(5) $\mathfrak{r}_{\mathbb{Q}}<\mathfrak{i}$,

(6) $\max \{\operatorname{cof}(\mathcal{M}), \mathfrak{r}\}<\mathfrak{r}_{\mathbb{Q}}$. 
4. The algebra $\mathcal{P}(\mathbb{Q}) /$ nwd and its relation to Dense $(\mathbb{Q})$. The Boolean algebra $\mathcal{P}(\mathbb{Q}) /$ nwd has been recently considered in the literature (see for example $[\mathrm{St}]$ and $[\mathrm{FS}]$ ) and fits into the extensive study of analytic quotients by I. Farah $[\mathrm{Fa}]$. We will show that it is closely related to both Dense $(\mathbb{Q})$ and the Cohen forcing. Cohen forcing $\mathbb{C}$ will be viewed as the partial order $\left(2^{<\omega}, \supseteq\right)$. The algebra $\mathcal{P}(\mathbb{Q}) /$ nwd is equivalent, as a forcing notion, to the pre-order $(\mathcal{P}(\mathbb{Q}) \backslash$ nwd, $\subseteq$ nwd $)$. Just like Dense $(\mathbb{Q})$, the algebra $\mathcal{P}(\mathbb{Q}) /$ nwd is homogeneous and has $\mathfrak{c}^{+}$-cc but not $\mathfrak{c}$-cc. Unlike Dense $(\mathbb{Q}), \mathcal{P}(\mathbb{Q}) /$ nwd does not contain a dense $\sigma$-closed part; in fact, it adds new reals. We will show that all reals added by $\mathcal{P}(\mathbb{Q}) /$ nwd are Cohen over the ground model.

TheOREM 4.1. The forcing $\mathcal{P}(\mathbb{Q}) /$ nwd is proper and adds only Cohen reals.

Proof. To see that $\mathcal{P}(\mathbb{Q}) /$ nwd is proper, fix a countable elementary submodel $M$ of some large enough $H(\theta)$ and let $C \in \mathcal{P}(\mathbb{Q}) / \operatorname{nwd} \cap M$. Without loss of generality we can assume that $M$ is the union of an increasing $\in$-chain of elementary submodels $M_{n}$. Fix an enumeration $\left\{\mathcal{A}_{n}: n \in \omega\right\}$ of all maximal antichains in $M$ such that $\mathcal{A}_{n} \in M_{n}$. Recursively construct a sequence $\left\{D_{n}: n \in \omega\right\}$ in $\mathcal{P}(\mathbb{Q}) \backslash$ nwd so that

(1) $D_{0}=C$,

(2) $D_{n+1}$ is a dense subset of $D_{n}$,

(3) $D_{n} \in M_{n}$,

(4) $\left\{A \cap D_{n+1}: A \in \mathcal{A}_{n} \cap M_{n}\right.$ and $A \cap D_{n+1}$ is somewhere dense $\}$ is a maximal pairwise disjoint family of subsets of $D_{n}$ which differ from an open set by a nowhere dense set.

Having constructed $D_{n}$, construct $D_{n+1}$ as follows: Enumerate (in $M_{n+1}$ ) $\mathcal{A}_{n} \cap M_{n}$ as $\left\{A_{i}: i \in \omega\right\}$. Let

$$
D_{n+1}=\bigcup_{k \in \omega} B_{k}
$$

where

$$
\begin{aligned}
& B_{0}=\operatorname{int}\left(\overline{D_{n} \cap A_{0}}\right) \cap\left(D_{n} \cap A_{0}\right), \\
& B_{k}=\operatorname{int}\left(\overline{D_{n} \cap A_{k}}\right) \cap D_{n} \cap A_{k} \backslash \bigcup_{j<k} \bar{B}_{j} .
\end{aligned}
$$

Note that $D_{n+1} \in M_{n+1}$, as its definition only uses elements of $M_{n+1}$. As $D_{n}$ and $\mathcal{A}_{n}$ are both elements of $M_{n}$ and since $M_{n}=" \mathcal{A}_{n}$ is a maximal antichain", for every $U_{n} \subseteq \bar{D}_{n}$ there is an $A \in \mathcal{A}_{n} \cap M_{n}$ such that $D_{n} \cap A \cap U_{n}$ is not nowhere dense in $U_{n}$. So $D_{n+1}$ is a dense subset of $D_{n}$ and $\left\{A \cap D_{n+1}\right.$ : $A \in \mathcal{A}_{n} \cap M_{n}$ and $A \cap D_{n+1}$ is somewhere dense $\}$ is a maximal pairwise disjoint family of subsets of $D_{n}$ which differ from an open set (in $D_{n+1}$ ) by a nowhere dense set. 
Having constructed the sequence $\left\{D_{n}: n \in \omega\right\}$ use Proposition 2.2(ii) to find a set $D$ dense in $C$ which is $\subseteq^{*}$-included in all $D_{n}$. The fact that $D$ (viewed as a condition in $\mathcal{P}(\mathbb{Q}) /$ nwd) is $(C, M)$-generic follows from condition (4). Much more is actually true. Not only are all antichains $\mathcal{A}_{n} \cap M$ predense below $D$ but also any maximal antichain of elements of $\bigcup_{n \in \omega} \mathcal{A}_{n} \cap M$ is "really" a maximal antichain below $D$, which translates directly into the fact that every real in the extension is contained in a smaller extension by Cohen forcing.

One of the cardinal invariants of the Boolean algebra $\mathcal{P}(\mathbb{Q}) /$ nwd was considered by J. Steprāns. In [St] he showed that it is relatively consistent with ZFC that there is an uncountable maximal antichain in $\mathcal{P}(\mathbb{Q}) / \mathrm{nwd}$ of cardinality strictly less than $\mathfrak{b}$. Other cardinal invariants $(\mathfrak{p}(\mathcal{P}(\mathbb{Q}) /$ nwd), $\mathfrak{t}(\mathcal{P}(\mathbb{Q}) / \mathrm{nwd}), \mathfrak{h}(\mathcal{P}(\mathbb{Q}) / \mathrm{nwd})$ and $\mathfrak{s}(\mathcal{P}(\mathbb{Q}) /$ nwd $))$ are equal to $\omega$, due to the fact that the Cohen algebra regularly embeds into $\mathcal{P}(\mathbb{Q}) / \mathrm{nwd}$. On the other hand, the cardinal invariants $\mathfrak{r}(\mathcal{P}(\mathbb{Q}) /$ nwd) and $\mathfrak{i}(\mathcal{P}(\mathbb{Q}) /$ nwd) are of interest and are, in fact, equal to their Dense $(\mathbb{Q})$ analogues. In some sense this justifies calling $\mathfrak{r}_{\mathbb{Q}}$ a reaping number.

Proposition 4.2. $\mathfrak{r}(\mathcal{P}(\mathbb{Q}) / \mathrm{nwd})=\mathfrak{r}_{\mathbb{Q}}$ and $\mathfrak{i}(\mathcal{P}(\mathbb{Q}) / \mathrm{nwd})=\mathfrak{i}_{\mathbb{Q}}$.

Proof. To see that $\mathfrak{r}(\mathcal{P}(\mathbb{Q}) /$ nwd $) \leq \mathfrak{r}_{\mathbb{Q}}$ note that if $\mathcal{R}$ is a dense-reaping family then $\left\{R \cap U_{n}: R \in \mathcal{R}\right.$ and $\left.n \in \omega\right\}$ is a reaping family in $\mathcal{P}(\mathbb{Q}) /$ nwd, for if $X \in \mathcal{P}(\mathbb{Q}) \backslash$ nwd is not dense, then there is an $n \in \omega$ such that $U_{n} \cap X=\emptyset$, and if it is dense then, as $\mathcal{R}$ is dense-reaping, there are $R \in \mathcal{R}$ and $n \in \omega$ such that $X \cap R \cap U_{n}=\emptyset$ or $R \cap U_{n} \subseteq D$.

For the other direction, fix $\kappa<\mathfrak{r}_{\mathbb{Q}}$ and a family $\mathcal{R} \subseteq \mathcal{P}(\mathbb{Q}) \backslash$ nwd. For $R \in \mathcal{R}$ put $D_{R}=R \cup(\mathbb{Q} \backslash \bar{R})$. As $\kappa<\mathfrak{r}_{\mathbb{Q}}$, the family $\left\{D_{R}: R \in \mathcal{R}\right\}$ is not dense-reaping; hence there is a $D \in \operatorname{Dense}(\mathbb{Q})$ which splits all $D_{R}$ into two dense sets and in particular it splits each $R$ into two somewhere dense sets. So $\mathcal{R}$ is not reaping in $\mathcal{P}(\mathbb{Q}) /$ nwd and therefore $\kappa<\mathfrak{r}(\mathcal{P}(\mathbb{Q}) /$ nwd).

The proof of $\mathfrak{i}(\mathcal{P}(\mathbb{Q}) / \mathrm{nwd}) \leq \mathfrak{i}_{\mathbb{Q}}(=\mathfrak{i})$ is virtually identical to the proof of Proposition 2.6. Identify $\mathbb{Q}$ with a countable dense subset of $2^{\omega}$ and let $J_{n}=\{f \in \mathbb{Q}: f(n)=1\}$. The family $\left\{J_{n}: n \in \omega\right\}$ forms a subbase of the topology of $\mathbb{Q}$. Let $\mathcal{I}$ be a maximal dense-independent family of size $\mathfrak{i}_{\mathbb{Q}}$. Then $\mathcal{J}=\mathcal{I} \cup\left\{J_{n}: n \in \omega\right\}$ is an independent family in $\mathcal{P}(\mathbb{Q}) /$ nwd. Maximality of $\mathcal{J}$ follows easily from dense-maximality of $\mathcal{I}$ and the fact that any set independent of all $J_{n}$ is dense. So $\mathfrak{i}(\mathcal{P}(\mathbb{Q}) /$ nwd $) \leq \mathfrak{i}_{\mathbb{Q}}$.

Put $\kappa=\mathfrak{i}(\mathcal{P}(\mathbb{Q}) /$ nwd $)$. In order to show that $\kappa \geq \mathfrak{i}$, let $\mathcal{I}=\left\{\left[I_{\alpha}\right]: \alpha<\kappa\right\}$ be a maximal independent family in $\mathcal{P}(\mathbb{Q}) /$ nwd and consider a cofinal family in nwd indexed as $\left\{C_{\alpha}: \alpha<\kappa\right\}$, where each element appears infinitely often. There exists such a cofinal family because $\operatorname{cof}(\mathrm{nwd})=\operatorname{cof}(\mathcal{M}) \leq \mathfrak{r}_{\mathbb{Q}}$ and $\mathfrak{r}_{\mathbb{Q}} \leq \mathfrak{i}(\mathcal{P}(\mathbb{Q}) /$ nwd $)$. 
For each $\alpha<\kappa$, let $J_{\alpha}=I_{\alpha} \backslash C_{\alpha}$. Then $\mathcal{J}=\left\{J_{\alpha}: \alpha<\kappa\right\}$ is an independent family of subsets of $\mathbb{Q}$ since $\mathcal{I}$ is an independent family in $\mathcal{P}(\mathbb{Q}) /$ nwd. Moreover, if $A$ is any subset of $\mathbb{Q}$, there exist finite sets $F, G \subseteq \kappa$ such that $A \cap\left(\bigcap\left\{I_{\alpha}: \alpha \in F\right\} \backslash \bigcup\left\{I_{\alpha}: \alpha \in G\right\}\right)$ is a nowhere dense subset of $\mathbb{Q}$ and therefore is contained in $C_{\beta}$ for some $\beta \in \kappa \backslash(F \cup G)$ (as each $C$ appears infinitely often). Thus

$$
A \cap\left(\bigcap\left\{J_{\alpha}: \alpha \in F \cup\{\beta\}\right\} \backslash \bigcup\left\{J_{\alpha}: \alpha \in G\right\}\right)=\emptyset,
$$

and hence $\mathcal{J}$ is maximal independent.

A natural question concerning $\mathcal{P}(\mathbb{Q}) /$ nwd is the following:

Question 4.3. Does $\mathcal{P}(\mathbb{Q}) /$ nwd collapse $\mathfrak{c}$ to $\mathfrak{h}_{\mathbb{Q}}$ ?

Acknowledgments. The work on this paper was initiated during the third author's visit at the Center for Theoretical Study of the Charles University and completed during the subsequent stay of the first author at the Instituto de Matemáticas de la UNAM, Unidad Morelia. The authors wish to thank both institutions for their hospitality.

\section{References}

[BPS] B. Balcar, J. Pelant and P. Simon, The space of ultrafilters on $N$ covered by nowhere dense sets, Fund. Math. 110 (1980), 11-24.

[BS] B. Balcar and P. Simon, Disjoint refinement, in: J. D. Monk and R. Bonnet (eds.), Handbook of Boolean Algebras, Vol. 2, 1989, 333-386.

[BJ] T. Bartoszyński and H. Judah, Set Theory. On the Structure of the Real Line, A K Peters, 1995.

[Bl] A. Blass, Combinatorial cardinal characteristics of the continuum, in: Handbook of Set Theory, to appear.

[Ci] J. Cichon, On dense subsets of rational numbers, Acta Univ. Carolin. Math. Phys. 42 (2001), 5-10.

[Do] P. L. Dordal, A model in which the base-matrix tree cannot have cofinal branches, J. Symbolic Logic 52 (1987), 651-664.

[Fa] I. Farah, Analytic quotients, Mem. Amer. Math. Soc. 148 (2000), no. 702.

[FS] I. Farah and S. Solecki, Two $F_{\sigma \delta}$ ideals, Proc. Amer. Math. Soc. 131 (2003), 1971-1975.

[Fr] D. H. Fremlin, The partially ordered sets of the measure theory and Tukey's ordering, Note Mat. 11 (1991), 177-214.

[Je] T. Jech, Set Theory, The Third Millennium Edition, revised and expanded, Springer, Berlin, 2003.

[KW] A. Kamburelis and B. Węglorz, Splittings, Arch. Math. Logic 35 (1996), 263-277.

[Ke] K. Keremedis, On the covering and the additivity number of the real line, Proc. Amer. Math. Soc. 123 (1995), 1583-1590.

[Ku] K. Kunen, Set Theory. An Introduction to Independence Proofs, North-Holland, Amsterdam, 1980. 
[Kr] K. Kuratowski, Introduction to Set Theory and Topology, PWN-Polish Sci. Publ., Warszawa, 1977.

[Mi] A. Miller, Rational perfect set forcing, in: J. Baumgartner, D. A. Martin, and S. Shelah (eds.), Axiomatic Set Theory, Contemp. Math. 31, Amer. Math. Soc., 1984, 143-159.

[MHD] J. Moore, M. Hrušák and M. Džamonja, Parametrized $\diamond$-principles, Trans. Amer. Math. Soc. 356 (2004), 2281-2306.

[Sh] S. Shelah, Proper and Improper Forcing, Springer, 1998.

[SS] S. Shelah and O. Spinas, The distributivity numbers of $\mathcal{P}(\omega) /$ fin and its square, Trans. Amer. Math. Soc. 352 (2000), 2023-2047.

[St] J. Steprāns, The cardinal invariant $\mathfrak{a}$ in the quotient algebra of the rationals modulo the nowhere dense subsets, Real Anal. Exchange 27 (2001/02), 795-800.

[Va] J. Vaughan, Small uncountable cardinals and topology, in: J. van Mill and G. M. Reed (eds.), Open Problems in Topology, North-Holland, 1990, 196-216.

Mathematical Institute

Instituto de Matemáticas

of the Academy of Sciences of the Czech Republic

UNAM Unidad Morelia

Žitná 25

A. P. 61-3, Xangari

11567 Praha 1, Czech Republic

E-mail: balcar@cts.cuni.cz

C.P. 58089, Morelia, Mich., México

E-mail: fernando@matmor.unam.mx michael@matmor.unam.mx

Received 2 July 2004;

in revised form 17 August 2004 